\title{
AUFSÄTZE
}

\section{Wahlrecht 2013 mit Schattenseiten. Oder: Ein kritisches Lob dem vom Bundesverfassungsgericht verworfenen Bundeswahlgesetz von 2011*}

\author{
Christian Hesse
}

Mit dem Urteil vom 3. Juli 2008 hat das Bundesverfassungsgericht das zuvor geltende Wahlrecht (in der Folge als BWahlG 2008 bezeichnet) zum Deutschen Bundestag in Teilen für verfassungswidrig erklärt und den Gesetzgeber verpflichtet, eine verfassungsgemäße Neuregelung vorzunehmen. Konkreter Anlass war die als „Negatives Stimmgewicht“ bezeichnete Paradoxie, die den Sachverhalt beschreibt, dass unter bestimmten Konstellationen ein Zuwachs an Zweitstimmen zu einem Verlust an Sitzen der Landeslisten führen kann oder umgekehrt. Dies war bei einer Nachwahl zur Bundestagswahl 2005 in Dresden geschehen. ${ }^{1}$ Als Reaktion auf den Regelungsauftrag beschloss der Bundestag am 29. September 2011 Änderungen der $\$ \$ 6$ und 7 des Bundeswahlgesetzes (Bundesgesetzblatt 2011 Teil I S. 2313, in der Folge als BWahlG 2011 bezeichnet). Mit Urteil vom 25. Juli 2012 erklärte das BVerfG auch dieses Wahlgesetz für verfassungswidrig ${ }^{2}$, was den Bundestag erneut zwang, das Wahlrecht anzupassen. Dies geschah mit dem Gesetzesbeschluss vom 21. Februar 2013, dem alle Fraktionen (außer Die Linke) zustimmten. ${ }^{3}$ Dieser Beitrag befasst sich mit den Eigenschaften des ursprünglichen BWahlG 2011 und mit den im Bundestag diskutierten Alternativmodellen aus wahlmathematischer Perspektive. Ziel ist es, die Stärken und Schwächen der verschiedenen Gesetzentwürfe für sich und im Vergleich herauszuarbeiten. Auf dieser Grundlage kann auch eine erste Einschätzung des nunmehr geltenden Rechts erfolgen.

\section{Auswirkungen des BWablG 2011}

Das am 29. September 2011 vom Bundestag verabschiedete Wahlrecht ${ }^{4}$ sah folgendes Verfahren vor: Zunächst wird jedem Wahlkreissieger ein Direktmandat zugeteilt. Die Auswer-

* Dieser Beitrag basiert auf einem Gutachten, das der Verfasser auf Anfrage der CDU/CSU-Fraktion des Bundestages zum 26. Februar 2012 erstellt hat und dessen Ergebnisse er bei der mündlichen Verhandlung des Bundesverfassungsgericht am 5. Juni 2012 in Sachen Verfassungsklage gegen das Wahlrecht in Teilen vorgetragen hat.

1 Zum BVerfG-Urteil 2008 vgl. Dieter Nohlen, Erfolgswertgleichheit als fixe Idee oder: Zurück zu Weimar? Zum Urteil des Bundesverfassungsgerichts über das Bundeswahlgesetz vom 3. Juli 2008, in: ZParl, 40. Jg. (2009), H. 1, S. 179 - 195. Zur Dresdner-Vorgeschichte vgl. Joachim Behnke, Strategisches Wählen bei der Nachwahl in Dresden zur Bundestagswahl 2005, in: PVS, 49. Jg. (2008), H. 4, S. 695 - 720; Franz Urban Pappi / Michael Herrmann, Überhangmandate ohne negatives Stimmgewicht: Machbarkeit, Wirkungen, Beurteilung, in: ZParl, 41. Jg. (2010), H. 2, S. $260-278$, S. 261.

2 BVerfG, 2 BVF 3/11 vom 25. Juli 2012, http://www.bverfg.de/entscheidungen/ fs20120725_2bvf000311.html (Abruf am 18. Februar 2013).

3 Vgl. Günter Bannas, Neues Wahlrecht ist beschlossen, in: FAZ vom 22. Februar 2013, S. 2.

4 BT-Drucksache 17/6290 in der Fassung des Buchstaben a der Beschlussempfehlung des Innenausschusses, BT-Drucksache 17/7069. 
tung der Zweitstimmen erfolgt anschließend in drei Schritten. In einem ersten Schritt wird die reguläre Gesamtzahl der Sitze im Bundestag zunächst auf die einzelnen Länder verteilt. Bemessungsgröße hierfür ist die Gesamtzahl der Wähler in jedem Bundesland.

In einem zweiten Schritt werden in jedem Bundesland die nach Schritt 1 ermittelten Sitze mit Sainte-Laguë/Schepers an die Landeslisten der zuteilungsberechtigten Parteien weitergegeben. Bemessungsgröße hierfür sind die von den Parteien im jeweiligen Land erzielten Zweitstimmen.

In einem dritten Schritt werden weitere Mandate vergeben, hier als Reststimmen-Mandate bezeichnet. Um deren Anzahl parteispezifisch zu ermitteln wird zunächst in jedem Land der Landes-Hare-Quotient ${ }^{5}$ errechnet. Als (positive) Reststimmen einer Partei sind jene Zweitstimmen für die Partei in einem Land definiert, die über das Produkt aus dem LandesHare-Quotienten und der Anzahl der nach Sainte-Laguë/Schepers errechneten Sitze hinausgehen. Diese Reststimmen werden für jede Partei und jedes Bundesland erhoben, auf Bundesebene summiert, sodann durch den Bundes-Hare-Quotienten ${ }^{6}$ dividiert und kaufmännisch gerundet. Der gerundete Wert ist die Anzahl der auf die jeweilige Partei entfallenden Reststimmen-Mandate. Die Reststimmen-Mandate einer Partei werden an die Landeslisten der Partei zuerst in der Reihenfolge der jeweils höchsten (noch verbleibenden) Überhänge vergeben ${ }^{7}$ und dann in der Reihenfolge der höchsten Reststimmenzahlen.

Um die Auswirkungen dieses Wahlgesetzes einschätzen zu können, soll zunächst ermittelt werden, inwieweit es gegenüber dem BWahlG 2008 zu einer anderen Sitzverteilung im Bundestag geführt hätte (vgl. Tabelle 1).

Wie erkennbar, sind die Änderungen in der Sitzzuweisung bei jeder einzelnen Wahl sowie auch für jede Partei über alle Wahlen kumuliert jeweils gering. Insofern kann das BWahlG 2011 bezüglich der resultierenden Ergebnisse als minimal-invasiv bewertet werden.

Generell sollte bei der Einschätzung eines neuen Sitzzuteilungsmodells hinsichtlich des Grades seiner Invasivität in Relation zu dem bis Dezember 2011 gültigen Modell der Blick auf die bewirkten Änderungen bei der praktischen Umsetzung gelenkt werden. Das Mischsystem des personalisierten Verhältniswahlrechts, wie es in der Bundesrepublik mit leichten Modifikationen seit Einführung des Systems der Zweitstimmen 1953 in Gebrauch ist, stößt auf breite Zustimmung in der Bevölkerung, ist verfassungspolitisch bewährt, wird mehrheitlich als erhaltenswert angesehen, wurde in der nationalen und internationalen Literatur meistenteils gelobt und diente andernorts ${ }^{8}$ bereits als Vorbild. Insofern gebührt einer ergebnisseitig gering-invasiven, weitgehend strukturerhaltenden Modifikation unter Abbau der vom BVerfG als verfassungswidrig monierten Aspekte der Vorzug gegenüber fundamentaleren Neukonstruktionen.

Jedes Sitzzuteilungsverfahren operiert in einem Umfeld von möglichen Wechselwirkungen zwischen derzeit sechs im Bundestag vertretenen Parteien, die um Erst- und Zweitstim-

5 Der Landes-Hare-Quotient ist die im jeweiligen Land für einen Sitz durchschnittlich erforderliche Zweitstimmenzahl. Der Wert errechnet sich als Quotient der zuteilungsfähigen Zweitstimmen in einem Land geteilt durch die Zahl der im jeweiligen Land zu vergebenden Sitze.

6 Der Bundes-Hare-Quotient ist die im ganzen Wahlgebiet für einen Sitz durchschnittlich erforderliche Zweitstimmenzahl. Der Wert errechnet sich als Quotient aller zuteilungsfähigen Zweitstimmen auf Bundesebene geteilt durch die Gesamtzahl der zu vergebenden Mandate auf Bundesebene.

7 Die Reststimmen-Mandate werden mit den Überhangmandaten verrechnet, erstere werden also nicht zusätzlich vergeben.

81996 führte Neuseeland bei Parlamentswahlen ein Zweistimmen-Wahlsystem deutschen Typs ein. 


\begin{tabular}{|c|c|c|c|c|c|c|c|c|}
\hline & हु & 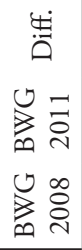 & 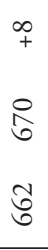 & 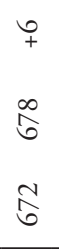 & 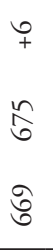 & 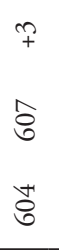 & 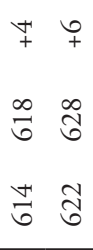 & 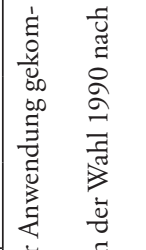 \\
\hline & 美 & 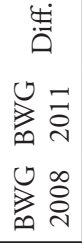 & $\begin{array}{l}{ }_{+1} \\
\simeq \\
\simeq\end{array}$ & $\begin{array}{l}+ \\
\tilde{m} \\
\stackrel{n}{\infty}\end{array}$ & $\begin{array}{l}\stackrel{m}{+} \\
\hat{m}\end{array}$ & $\begin{array}{l}0 \\
+1 \\
N\end{array}$ & 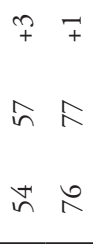 & 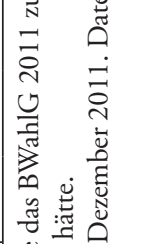 \\
\hline & : & 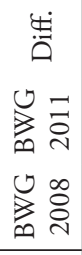 & $\begin{array}{l}\overrightarrow{1} \\
\infty\end{array}$ & $\begin{array}{l}\stackrel{+}{+} \\
\stackrel{n}{n} \\
\stackrel{F}{q}\end{array}$ & $\begin{array}{l}\stackrel{+}{+} \\
\stackrel{F}{F} \\
\stackrel{F}{\curvearrowright}\end{array}$ & $\begin{array}{l}\stackrel{+}{+} \\
i \\
i n\end{array}$ & $\begin{array}{l}\stackrel{F}{+} \\
\stackrel{n}{R} \\
\ddot{n} \infty\end{array}$ & 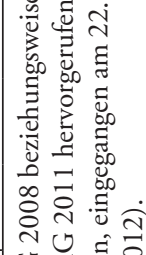 \\
\hline 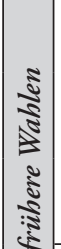 & 官 & 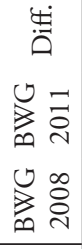 & $\begin{array}{l}\stackrel{+}{+} \\
\infty\end{array}$ & \begin{tabular}{l}
$\mp$ \\
$\stackrel{\infty}{+}$ \\
\multirow{+}{F}{}
\end{tabular} & $\begin{array}{l}\stackrel{\sim}{+} \\
\stackrel{n}{\forall}\end{array}$ & $\begin{array}{l}\stackrel{+}{+} \\
\stackrel{\leftrightarrow}{q}\end{array}$ & $\begin{array}{l}\mp \stackrel{+}{\sigma} \\
\sigma \approx \\
\sigma \approx\end{array}$ & 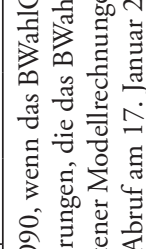 \\
\hline $\begin{array}{c}5 \\
\frac{2}{2} \\
5 \\
5 \\
5 \\
5 \\
5 \\
5 \\
5 \\
5\end{array}$ & 2 & 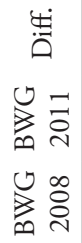 & $\begin{array}{l}\stackrel{\sim}{+} \\
\stackrel{n}{n}\end{array}$ & $\begin{array}{l}F \\
\ddot{n} \\
\stackrel{n}{n}\end{array}$ & $\widehat{F}$ & $\begin{array}{l}p \\
n\end{array}$ & 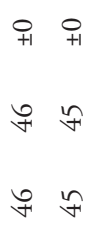 & 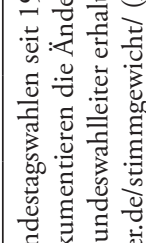 \\
\hline 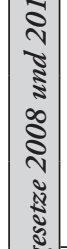 & ? & 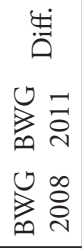 & 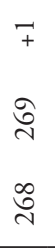 & 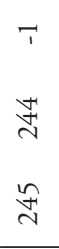 & 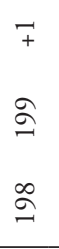 & $\begin{array}{l}\stackrel{+}{ } \\
\stackrel{\Xi}{\Xi} \\
\check{\Xi}\end{array}$ & 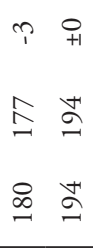 & 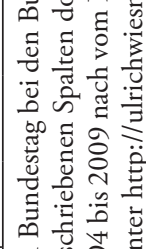 \\
\hline 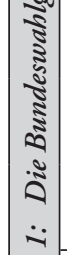 & $\stackrel{\hat{\omega}}{\vec{\omega}}$ & 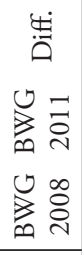 & $\begin{array}{l}\stackrel{\sim}{\sim} \\
\infty \\
\stackrel{\sim}{\sim}\end{array}$ & 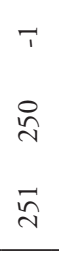 & $\begin{array}{l}\stackrel{\searrow}{ } \\
\infty \\
\curvearrowright\end{array}$ & $\begin{array}{l}\text { + } \\
\vec{a} \\
\vec{a} \\
\vec{a}\end{array}$ & 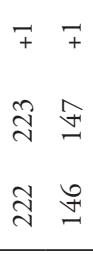 & 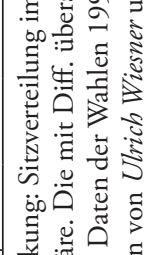 \\
\hline छัँ & & 敢 & $\stackrel{2}{\varrho}$ & 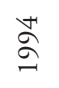 & $\stackrel{\infty}{\curvearrowright}$ & 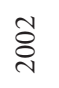 & 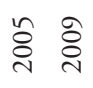 & 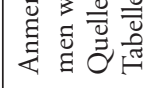 \\
\hline
\end{tabular}


men in 16 Bundesländern konkurrieren. Es sollte Mandate an Kandidaten, Parteien und auf Länder verteilen unter hinreichender Wahrung beziehungsweise nicht entscheidender Verletzung einer Reihe von Fairness-, Konsistenz-, Repräsentations- und Proporz-Bedingungen und weitgehender Vermeidung einer Reihe von Wahlparadoxien. ${ }^{9}$ Vor dem Hintergrund dieser Optimalitätskriterien ist die Sitzzuteilung beim Mischsystem der personalisierten Verhältniswahl eine echte Herausforderung. Mehr noch: Mathematische Unmöglichkeitstheoreme verhindern die Existenz eines Verfahrens, das völlig makellos ist.

\section{Bewertung des BWablG 2011}

\subsection{Ländersitzkontingente}

Der strukturell gravierendste Wandel im BWahlG 2011 ist die Umkehrung von Oberzuteilung und Unterzuteilung. So werden Sitze zunächst auf die einzelnen Länder verteilt, und zwar nach Maßgabe der Zahl der abgegebenen Wahlzettel in den Ländern. Diese Handhabung führt gegenüber einer möglichen Berechnung der Sitzkontingente nach zuteilungsberechtigten Zweitstimmen oder nach der Bevölkerungszahl, wie aus Gründen der Systemlogik in manchen Stellungnahmen vorgeschlagen wurde ${ }^{10}$, zu nur geringen Veränderungen bei der Sitzverteilung.

Ein Vergleich der Sitzkontingente auf Basis der Wahlbeteiligung statt nach Zweitstimmen zeigt: In elf von 16 Ländern sind keine Änderungen zu verzeichnen, in drei Ländern hätte sich eine Veränderung um einen Sitz in positive oder negative Richtung ergeben und in den beiden größten Ländern Bayern und Nordrhein-Westfalen liegen die bewirkten Änderungen bei plus drei beziehungsweise minus zwei Sitzen. Insbesondere die Sitzzuteilungen in den fünf neuen Ländern blieben untangiert. Zwar ist die Wahlbeteiligung dort seit der Bundestagswahl 1990 ausgeprägt geringer, doch gibt es eine hohe Korrelation zwischen der Zahl der abgegebenen Stimmzettel und der Zahl der abgegebenen Zweitstimmen. Aus diesen und anderen Gründen wirkt sich die Festlegung der Wählerzahl als Bemessungsgröße für die Ländersitzkontingente gegenüber einer fiktiven Bemessung nach Zweitstimmen nicht negativ für die neuen Bundesländer aus.

Ein Vergleich der Ländersitzkontingente, die bei Bemessung nach Wählerzahl statt nach Bevölkerungszahl als Referenzgröße für die Oberzuteilung bei der Bundestagswahl 2009 erwachsen, zeigt: In sechs Ländern sind keine Änderungen bei der Zahl der zugeteilten Sitze zu verzeichnen, in sieben Ländern gibt es Veränderungen um einen Sitz in positive oder negative Richtung, in Nordrhein-Westfalen um minus zwei, in Niedersachsen von plus drei, und in Baden-Württemberg würde eine Veränderung um minus vier eintreten. Auch hier ergibt sich der Befund, dass die Ansetzung der Wählerzahl zur Ermittlung der Ländersitzkontingente statt der Bevölkerungszahl sich für die fünf neuen Bundesländer nicht negativ

9 Für mögliche Paradoxien in Wahlsystemen siehe Hannu Nurmi, Voting Paradoxes - And How To Deal With Them, Berlin 1999.

10 Siehe etwa Hans Meyer, Normenkontrollantrag gegen die vom Bundestag am 29. September 2011 beschlossenen Änderungen der $\$ \$ 6$ und 7 des Bundeswahlgesetzes (BGBl S. 2313), 2 BvF3/11. Darin auf Seite 5: „Außerdem ist die Bestimmung der Sitzkontingente jedes Landes nach der Wahlbeteiligung statt nach der Bevölkerungszahl inkonsequent.“ 
auswirkt, im Gegenteil: Brandenburg und Thüringen könnten je ein zusätzliches Mandat beanspruchen.

Geht man einen Schritt weiter und ermittelt die von den Änderungen bei den Ländersitzkontingenten ausgelösten Neuerungen in der Unterverteilung für die Landeslisten der Parteien, so profitiert bei Zugrundelegung der Wahlbeteiligung im Vergleich mit der Bemessung nach Zweitstimmen über alle Länder betrachtet die SPD mit einem zusätzlichen Sitz (Bayern); die CDU verliert zwei Mandate (Nordrhein-Westfalen, Schleswig-Holstein), CSU und FDP gewinnen jeweils einen Sitz (Bayern), so auch die Grünen (Berlin). Die Linke verliert zwei Mandate (Niedersachsen, Nordrhein-Westfalen).

Relativ zur Berechnung der Ländersitzkontingente nach Bevölkerungszahl ergeben sich bei Verwendung der Wählerzahl folgende Änderungen: Die SPD gewinnt zweimal je einen Sitz (Niedersachsen, Rheinland-Pfalz) und verliert zweimal je einen Sitz (Baden-Württemberg, Hamburg). Die CDU gewinnt zweimal je ein Mandat (Niedersachsen, Thüringen) und verliert zweimal je eines (Baden-Württemberg, Nordrhein-Westfalen). Die FDP gewinnt zweimal je einen Sitz (Niedersachsen, Schleswig-Holstein) und verliert einen (Baden-Württemberg). Bündnis90/Die Grünen gewinnt ein (Saarland) und verliert ein Mandat (BadenWürttemberg). Die Linke gewinnt einen Sitz (Brandenburg) und verliert zweimal je einen (Berlin, Nordrhein-Westfalen).

Als denkbares Manko der Errechnung der Ländersitzzahlen nach Wahlbeteiligung ist eine verzerrende Wirkung auf die Erfolgswerte zu prüfen. Vergleicht man Erfolgswerte länderspezifisch, hier quantifiziert mittels der Anzahl der Mandate, die den Parteien für eine Million anzurechnender Zweitstimmen in einem Bundesland zugesprochen werden, so liegt für die Bundestagswahl 2009 bei Anwendung der Zweitstimmenzahlen für die Ländersitzkontingente der durchschnittliche Erfolgswert - gemittelt über alle Länder - bei 14,7 Sitzen pro einer Million Stimmen. ${ }^{11}$ Mit Ausnahme von Bremen, das einen Wert von 15,6 Sitzen aufweist, bewegen sich die Erfolgswerte der übrigen Länder im Bereich von 14,2 bis 14,9 Sitzen pro einer Million Stimmen.

Wäre stattdessen die Wahlbeteiligung als Referenzgröße verwendet worden, läge der durchschnittliche Erfolgswert ebenfalls bei einem Wert von 14,7. In elf Ländern blieben die Erfolgswerte unverändert, und die Werte aller Länder lägen im Bereich von 14,2 bis 15,1 Sitzen, wiederum mit Ausnahme von Bremen mit unveränderten 15,6 Sitzen pro einer Million Stimmen. Die maximale Änderung aller Erfolgswerte läge bei 4,4 Prozent. Die Änderungen sind damit nur geringfügig und insofern tolerabel.

Unter dem Blickwinkel der Nivellierung von Erfolgswertunterschieden ist der anzustrebende Idealzustand natürlich die absolute Erfolgswertgleichheit. Dieser Zustand ist aber in der Regel nicht herstellbar. Die diversen Sitzzuteilungsverfahren erreichen nur gute oder weniger gute Annäherungen an diesen Optimalzustand.

Das BVerfG hat in früherer Rechtsprechung ${ }^{12}$ die Ansicht vertreten, dass zur Umsetzung von Verfassungsprinzipien geringfügige Erfolgswertunterschiede in Kauf genommen werden dürfen. Weitere und bedeutendere Differenzierungen der Erfolgswerte als jene, die auf die

11 Eigene Berechnungen auf der Grundlage von Daten in Joachim Behnke, „Dies ist das Wahlsystem meines Missvergnügens“. Eine Kritik des Entwurfs der CDU/CSU und FDP für eine Reform des Wahlgesetzes, http://www.zeppelinuniversity.de/deutsch/lehrstuehle/politikwissenschaft/CDU_ Wahlreformentwurf.pdf (Abruf am 15. Februar 2012).

12 BVerfGE 95, S. 335 vom 10. April 1997. 
Verwendung der Wahlbeteiligung als Bemessungsgröße für die Ländersitzkontingente zurückzuführen sind, werden von Überhangmandaten erzeugt. Doch auch diese Verzerrungen werden in ihrer Tragweite bei Weitem dominiert von den erheblichen Erfolgswertunterschieden, die auf Parteienbasis von der (nach Rechtsprechung des BVerfG verfassungskonformen $^{13}$ ) Fünfprozentsperrklausel herbeigeführt werden.

Als Nachteil des im BWahlG 2011 verankerten Modus der Berechnung der Ländersitzzahlen ist zu benennen, dass die Wahlbeteiligung eine variable, erst mit dem abgeschlossenen Wahlvorgang festliegende Größe ist und als solche bei hypothetischer Änderung auch eine Variation bei den Ländersitzkontingenten nach sich ziehen kann. Die Implementierung einer nicht vom Wahlergebnis abhängigen Bemessungsgröße, wie der Bevölkerungszahl, hätte aber für das BWahlG 2011 - bliebe alles andere gleich - Nachteile hinsichtlich einer Verzerrung der Erfolgswerte mit sich gebracht. In dieser Situation wirken sich die stark variierenden Wahlbeteiligungen in den Ländern schließlich auch stark verzerrend aus: Die Wahlbeteiligung bei der Bundestagswahl 2009 war in Hessen mit 73,8 Prozent am höchsten und mit 60,5 Prozent am geringsten in Sachsen-Anhalt. Wenn Sitzkontingente an die Länder auf der Basis einer jeden von der Wahl selbst nicht beeinflussten konstanten Referenzgröße bestimmt worden wären, hätte eine Wählerstimme aus Sachsen-Anhalt gegenüber einer Wählerstimme aus Hessen einen rund 20 Prozent höheren Erfolgswert erzielt. Dieser Unterschied ist eklatant.

Jede vom Verhalten der Wahlberechtigten nicht beeinflusste Größe als Grundlage der Berechnung der Ländersitzzahlen, wie zum Beispiel die Zahl der Wahlberechtigten oder die Bevölkerung der Länder, hätte auf der vorgeschalteten Ebene der Sitzzuweisung an die Länder Bikausale Monotoniefehler vollständig vermieden. Dieser Begriff ist in Abgrenzung zum Begriff des Negativen Stimmgewichts (NSG) zu verstehen. NSG soll hier allein für die im Tenor unter Nr. 1 des BVerfG-Entscheids vom 3. Juli 2008 verwendete Definition reserviert werden, die an Variationen bei der Zweitstimmenzahl für eine Partei in einem Land anknüpft. Bikausale Monotoniefehler bedürfen dagegen zusätzlich einer Variation der Wählerzahlen, um nicht-monotone Wirkungen auf die Mandatszahlen auszuüben. Diese Effekte können im BWahlG 2011 auftreten.

Man kann dies durch ein Beispiel aus der Anwendung des BWahlG 2011 auf die Bundestagswahl von 2009 belegen: Wenn 12.523 Wähler der Linken in Bayern nicht zur Wahl gegangen wären, also mit der Zweitstimme auch ihre Erststimme entzogen hätten und alles andere gleich geblieben wäre, hätte Die Linke in Bayern nach wie vor sechs Sitze erhalten, das Land Bayern aber insgesamt einen Sitz verloren. Dieser wäre nach Nordrhein-Westfalen gewandert und dort zufällig wieder der Linken zugeteilt worden. Die Zahl der ReststimmenMandate bliebe unverändert. Im Ergebnis hätte Die Linke ein Mandat gewonnen.

Dieses Beispiel zeigt zudem, dass es im BWahlG 2011 im Unterschied zum BWahlG 2008 eine Variante von Bikausalem Monotoniefehler ${ }^{14}$ gibt, die nicht an Überhangmandate ge-

13 BVerfGE 82, S. 322 vom 29. September 1990.

14 Es liegt hier nicht NSG vor, sondern ein Bikausaler Monotoniefehler, da es sich nicht um den vom BVerfG im Tenor seines Urteils vom 3. Juli 2008 monierten Effekt handelt, der eine Variation lediglich der Zweitstimmen vorsieht, nicht aber die weitergehende Voraussetzung einer Ko-Variation der Wählerzahl expressis verbis erwähnt. Wird aber die Wählerzahl in Bayern konstant gehalten, kommt es bei der angesprochenen hypothetischen Reduktion der Zweitstimmenzahl für Die Linke nicht zu dem skizzierten Sitzgewinn und somit nicht zu NSG im Sinn des BVerfGUrteils. 
knüpft ist. Zudem verdeutlichen die vorstehenden Begriffsbildungen, dass eine sachgerechte Analyse des BWahlG 2011 anspruchsvoller ist als die des BWahlG 2008. Auch muss sie hinsichtlich der inversen mandatswirksamen Effekte differenzierter sein, als es in vielen früheren Kommentaren bedacht worden ist.

Theoretisch ist im BWahlG 2011 die Sachlage denkbar, dass sich unter der Annahme von einigen zusätzlichen, für eine Partei P in einem Bundesland B abgegebenen Zweitstimmen das Sitzkontingent von Land B auf Kosten eines Landes C um einen Sitz vergrößert. Im ungünstigsten Fall bei entsprechender Überhangsituation in beiden Ländern könnte der aus Land C abwandernde Sitz der Partei P verloren gehen und im Land B der rivalisierenden Partei Q zu Gute kommen. Dann hätten, wenn zusätzlich die dabei günstiger werdende Reststimmensituation sich nicht zugunsten von Partei P auswirkt, einige zusätzliche P-Wähler ihrer Partei doppelt geschadet. ${ }^{15}$

Eine für die Beurteilung von NSG relevante Eigenheit des BWahlG 2011 besteht ferner darin, dass unabhängig von NSG-Effekten eine geringfügige fiktive Abnahme der Wahlbeteiligung in einem Land dazu führen kann, dass auf Bundesebene insgesamt mehr Mandate vergeben werden. Der umgekehrte Effekt ist ebenso möglich. Die weitere Wirkung des obigen Fallbeispiels von 12.523 weniger Stimmen für Die Linke in Bayern bei der letzten Bundestagswahl besteht nämlich darin, dass auf Bundesebene bei Einbeziehung der Reststimmenverwertung zwei zusätzliche Mandate vergeben würden. ${ }^{16}$ Das zweite zusätzliche Mandat ginge an die SPD.

Das Bestehen dieser Möglichkeit, die im zuvor geltenden Wahlrecht nicht auftrat, hat Auswirkungen auf die Variation oder Nicht-Variation der Wählerzahlen bei Quantifizierungen der Intensität von NSG.

Es ergibt sich der Befund (siehe Abschnitt 3 für weitere Details), dass die Wahrscheinlichkeiten des Auftretens der hier untersuchten inversen Effekte entscheidend davon abhängen, ob allein die Zweitstimmenzahl einer Partei in einem Bundesland variiert wird bei Konstanthaltung der Wahlbeteiligungen in allen Ländern (NSG im Sinne des BVerfGE vom 3. Juli 2008), oder ob als weitergehende Einräumung eine Ko-Variation von Zweitstimmenzahl und Wählerzahl zugelassen wird (Bikausaler Monotoniefehler).

\section{2. Überhangmandate}

Das BVerfG hat in seinem Urteil vom 3. Juli 2008 nicht die Möglichkeit des Auftretens von Überhangmandaten im BWahlG 2008 moniert. Zudem hat es dem Gesetzgeber zur Behebung von NSG als eine von mehreren Alternativen einen Weg gewiesen, der nicht an der Beseitigung von Überhangmandaten ansetzt, sondern an der Trennung der Listenverbindungen der Parteien. Mit der Verabschiedung des BWahlG 2011 wurde vom Gesetzgeber der letztgenannte Weg beschritten.

15 Dieser Fall hat eine gewisse Analogie zum SPD-Modell (und generell zu Modellen, die Ausgleichsmandate für Überhangmandate vorsehen) und dessen theoretischer Möglichkeit eines doppelten Malus für eine Partei und deren Schwesterpartei oder Koalitionspartner bei fiktivem Verlust sowohl eines Überhangmandats als auch eines Ausgleichsmandats, siehe Abschnitt 4.1.

16 Ein drittes zusätzliches Reststimmen-Mandat erhält die CDU. Dieses wirkt sich aber wegen der CDU-Überhangmandate nicht mandatsvermehrend für die Partei aus. 
Überhangmandate sind unter anderem mit Argumenten kritisiert worden, die sich auf das Gebot der Erfolgswertgleichheit der Wählerstimmen berufen. Der Erfolgswert der Stimmen für eine Partei bei einer Wahl kann definiert werden als der Quotient aus der Mandatszahl dieser Partei geteilt durch die Gesamtzahl der für sie abgegebenen Stimmen. Der relative Erfolgswert der Stimmen für eine Partei ist dann der Quotient aus dem Mandatsanteil für diese Partei geteilt durch den Stimmenanteil dieser Partei.

Das Gebot der Erfolgswertgleichheit verlangt idealiter, dass alle relativen Erfolgswerte gleich 1 sind, oder - im Realfall -, dass die Abweichungen davon mathematisch minimal sind. Die Minimierung kann vollzogen werden für den maximalen Unterschiedsbetrag oder den mittleren absoluten Unterschiedsbetrag oder das Mittel der quadrierten Unterschiedsbeträge der einzelnen Erfolgswerte. Das letztgenannte Abweichungsmaß, welches unter mancherlei quantitativen Gesichtspunkten das vorteilhafteste ist, führt zum Zuteilungsverfahren nach Sainte-Laguë/Schepers. Insofern ist dieses auch im BWahlG 2011 zum Einsatz kommende Verfahren bestmöglich in Bezug auf eine globale Nivellierung von Erfolgswertunterschieden.

Eine erfolgswertbasierte Kritik an Überhangmandaten ist zwar zutreffend, doch wird eine Beurteilung eines personalisierten Verhältniswahlsystems auf der Basis nur von Erfolgswertgleichheit nicht allen zu bedenkenden Aspekten dieses komplexen Wahlsystems gerecht. Eine erstrangige Akzentuierung der Erfolgswertgleichheit resultiert in der Regel aus dem impliziten Grundsatz, dass die Hauptfunktion von Mandatszuteilungsverfahren in der Demokratie darin besteht, die Stimmenverteilung in der Wählerschaft so gut wie möglich 1:1 in der Sitzverteilung im Parlament abzubilden. Diese Denkweise des Postulats der punktgenauen Umsetzung von Wählerstimmenanteilen in Mandatsanteile ist indes in einem personalisierten Verhältniswahlrecht mit Erst- und Zweitstimme eindimensional, da Erfolgswertgleichheit auf der Basis der Zweitstimmenverteilung gemessen und bewertet wird und so einzig das lineare Vertretungsprinzip der Verhältniswahl zum Maßstab erhoben wird, noch dazu eine reine Verhältniswahl in einem einzigen nationalen Wahlkreis ohne Sperrklausel als Idealmaßstab herangezogen wird. ${ }^{17}$ In einem Wahlrecht mit Sperrklausel wird aber der gravierendste Effekt, der die Erfolgswerte differenzierend tangiert und deren markantes Auseinanderlaufen bedingt, von dieser Sperrklausel herbeigeführt. Der von den Überhangmandaten verursachte Verzerrungseffekt ist demgegenüber vergleichsweise marginal.

\subsection{Reststimmenverwertung}

Die Reststimmenverwertung des BWahlG 2011 bewirkt eine Verringerung der Überhangmandate, im Durchschnitt um etwa 25 Prozent, indem sie einige überhängende Mandate mit Zweitstimmen in Form von Reststimmen unterlegt. Auch mildert sie eine mit den Überhangmandaten verbundene Verzerrung des Proporzes, bei den letzten beiden Bundestagswahlen um etwa 20 Prozent. Ferner ist damit zu rechnen, dass die großen Parteien in der Regel zumindest einige Überhangmandate erzielen ${ }^{18}$, so dass ihre gesamte ReststimmenMandatszahl ihnen entweder gar nicht oder nur teilweise zu Gute kommt.

17 Siehe auch Franz Urban Pappi / Michael Herrmann, Überhangmandate ohne negatives Stimmgewicht: Machbarkeit, Wirkungen, Beurteilungen, in: ZParl, 41 Jg. (2010), H. 2, S. 260 - 278.

18 Wahl 1990: CDU 6 Überhangmandate, SPD 0; Wahl 1994: CDU 12, SPD 4; Wahl 1998: SPD 13, CDU 0; Wahl 2002: SPD 4, CDU 1; Wahl 2005: SPD 9, CDU 7; Wahl 2009: CDU 21, CSU 3, SPD 0. 
Tabelle 2: Reststimmen-Mandate und Überhangmandate bei Anwendung des BWahlG 2011

\begin{tabular}{|l|c|c|c|c|c|c|c|}
\hline \multirow{2}{*}{ Wahl } & \multicolumn{7}{|c|}{ Reststimmen-Mandate und Überhangmandate } \\
\cline { 2 - 8 } & SPD & CDU & CSU & FDP & Grüne & Linke & Gesamt \\
\hline 1994 & $3\{0\}(4)[1]$ & $2\{0\}(11)[9]$ & 0 & $4\{4\}$ & $1\{1\}$ & $2\{2\}$ & $12\{7\}[15)[10]$ \\
1998 & $2\{0\}(11)[9]$ & $2\{2\}(0)[0]$ & $1\{1\}$ & $2\{2\}$ & $1\{1\}$ & $2\{2\}$ & $10\{8\}(11)[9]$ \\
2002 & $2\{0\}(3)[1]$ & $3\{3\}(0)[0]$ & 0 & $2\{2\}$ & $1\{1\}$ & $0\{0\}$ & $8\{6\}(3)[1]$ \\
2005 & $1\{0\}(8)[7]$ & $3\{0\}(5)[2]$ & 0 & $3\{3\}$ & $2\{2\}$ & $2\{2\}$ & $11\{7\}(13)[9]$ \\
2009 & $2\{1\}(1)[0]$ & $2\{0\}(22)[20]$ & $0\{0\}(2)[2]$ & $1\{1\}$ & $1\{1\}$ & $2\{2\}$ & $8\{5\}(25)[22]$ \\
\hline
\end{tabular}

Anmerkung: BWahlG 2011 in fiktiver Anwendung auf die Ergebnisse der Bundestagswahlen 1994 bis 2009 (für 1990 lagen keine Daten vor). Einträge ohne Klammern geben die Zahl der ReststimmenMandate an. Geschwungene Klammern „\{ \{\}$^{“}$ enthalten die Anzahl der sich mandatsvermehrend auswirkenden Reststimmen-Mandate. Runde Klammern „( )“ enthalten die Anzahlen der Überhangmandate ohne Reststimmenverwertung, in eckigen Klammern „[ [“ ist die Zahl der verbleibenden Überhangmandate nach Reststimmenverwertung gegeben. Die Anzahl von 0 ist der Übersichtlichkeit halber teils weggelassen.

Quelle: Daten nach Modellrechnungen des Bundeswahlleiters und eigenen Berechnungen.

Insgesamt wären bei diesen fünf Bundestagswahlen 33 zusätzliche Sitze aufgrund von Reststimmenverwertung zustande gekommen, im Mittel also sechs bis sieben pro Wahl. Von der Reststimmenverwertung profitieren erkennbar die kleinen Parteien (vgl. Tabelle 2). Die Regelung hat kompensatorischen Charakter. Bei Anwendung dieses Wahlrechts ohne Reststimmenverwertung würde augenfällig folgendes Problem für kleine Parteien bestehen: Wenn ihre Zweitstimmenzahl nicht hinreichend ist, um in einem Land damit mindestens einen Sitz zu erzielen, sind diese Zweitstimmen für die Partei gänzlich verloren. Sie üben weder im betrachteten Land noch in irgendeinem anderen Land für die betroffene Partei eine positive Wirkung aus. Zwar ist kritisiert worden, dass diese Stimmen durch mögliche Vergrößerung des Sitzkontingents eines Landes einer anderen Partei zugute kommen können und dass es bei der Bundestagswahl 2009 über drei Millionen Stimmen gegeben hätte, der das BWahlG 2011 Mandatsgewicht für fremde Parteien gegeben hätte, doch wird bei dieser Kritik nicht bedacht, dass diese Effekte über alle Länder betrachtet nicht nur nicht additiv sind, sondern sich sogar neutralisieren, da derartige Stimmen mit Anzahlen von gleicher Größenordnung in allen Ländern anfallen.

Das skizzierte Problem der potentiell verlorenen Stimmen, dem sich Landesverbände kleiner Parteien generell gegenübersehen, wird durch das Phänomen der effektiven Sperrklausel verstärkt. Ursache dafür ist der Umstand, dass das einem kleinen Land zukommende Sitzkontingent so gering sein kann, dass erst ein höherer Prozentsatz der Stimmen als jener der gesetzlichen Sperrklausel von fünf Prozent in diesem Land für eine Partei zu einem gewonnenen Zweitstimmensitz führt. ${ }^{19}$ Dies erhöht die gesetzliche Fünfprozenthürde für eine Partei auf einen effektiven Wert, der weitaus höher liegen kann. ${ }^{20}$ Auch diesen Sachver-

19 Ein Beispiel aus dem BWahlG 2008: Trotz eines Zweitstimmenanteils von 8,4 Prozent in Bremen bei der Wahl 2005 blieb die Linke ohne Mandat in diesem Land.

20 Mathematisch hängt der Prozentsatz der effektiven Sperrklausel vom eingesetzten Zuteilungsverfahren, von der verfügbaren Mandatszahl M und der Parteienzahl P im Wahlgebiet ab. Für das Verfahren von Sainte-Laguë/Schepers ergibt sich (nach Friedrich Pukelsheim / Sebastian Maier I Peter Leutgäb, Zur Vollmandat-Sperrklausel im Kommunalwahlgesetz, in: Nordrhein-Westfälische 
halt mildert die Reststimmenverwertung. Zwar werden Reststimmen-Mandate nicht zielgenau an die Landeslisten vergeben, die von einer das gesetzliche Quorum überschreitenden effektiven Sperrwirkung betroffen sind, doch erfolgt diese Zuweisung wegen der erheblichen Reststimmenzahl dieser Landeslisten immerhin mit großer Wahrscheinlichkeit.

Das Divisorverfahren mit Standardrundung nach Sainte-Laguë/Schepers ist in der Theorie im Mittel unverzerrt und bevorzugt oder benachteiligt im Durchschnitt weder große noch kleine Parteien. Vor diesem Hintergrund ist die Reststimmenverwertung in Verbindung mit der Verwendung von Sainte-Laguë/Schepers als „Systembruch“21, als „wahlrechtliche(s) Kuriositätenkabinett" ${ }^{22}$ und „Absurdität“ 23 engagiert kritisiert worden. Doch erscheint die von der konkreten Ausgestaltung der Reststimmenverwertung de facto hervorgerufene Begünstigung kleiner Parteien bei der Vergabe von Reststimmen-Mandaten gerechtfertigt, um der effektiv über fünf Prozent liegenden Ausschluss-Schwelle entgegenzuwirken, der sich kleine Parteien in kleinen Ländern gegenübersehen.

Noch ein weiterer Gesichtspunkt kann zugunsten einer Reststimmenverwertung auch in Kombination mit dem nur im Mittel unverzerrten Verfahren von Sainte-Laguë/Schepers geltend gemacht werden: Wenn eine Landesliste einer Partei idealiter aufgrund der Zweitstimmen eigentlich ein Anrecht auf einen etwas größeren (nicht ganzzahligen) Anteil der Sitze hat als ihr nach Sainte-Laguë/Schepers in diesem Land (ganzzahlig) zugeteilt wird, gingen die diesen Bruchteilen entsprechenden Zweitstimmen bei einer Trennung der Listen, wie sie das BWahlG 2011 vorsieht, ebenfalls vollständig verloren. Bis auf einen Anteil, der weniger als einem halben Mandat entspricht, fanden im BWahlG 2008 alle in den Ländern nicht als Mandate zu Buche schlagenden Stimmen bei der Oberverteilung Berücksichtigung. Bei Verankerung der Länder als weitgehend separate Teilwahlgebiete ${ }^{24}$, wie sie das BWahlG 2011 vornimmt, ist dies nicht mehr der Fall. Zudem kann man von einem mathematischen Rundungsvorgang begünstigt oder benachteiligt werden. Im hierbei nachteiligsten Fall könnte eine Partei in vielen oder gar jedem der 16 Bundesländer durch Rundungspech den Zugewinn eines weiteren Mandats gerade knapp verpassen, also schlimmstenfalls in jedem der 16 Bundesländer nahezu ein halbes Mandat einbüßen. Auch diese schwerwiegende Möglichkeit kompensiert die Reststimmenverwertung.

Die konkrete Ausgestaltung der Reststimmenverwertung nach $\$ 6$ Abs. 2a, insbesondere der Sachverhalt, dass der bei der Berechnung zur Anwendung kommende Bundes-Hare-

Verwaltungsblätter 2009, S. 85 - 90) die Formel 1/(2M - P + 2). Setzt man für Bremen $M=5$ Sitze an bei $\mathrm{P}=5$ Parteien, so ergibt sich eine effektive Sperrklausel von $1 /(10-5+2)=1 / 7=$ 14,3 Prozent.

21 Friedrich Pukelsheim, Stellungnahme für die Anhörung des Innenausschusses des Deutschen Bundestages am 5. September 2011, Ausschuss-Drucksache 17(4) 327A, S. 24.

22 Hans Meyer, a.a.O. (Fn. 10), S. 16.

23 Joachim Behnke, a.a.O. (Fn. 11), S. 37. Bei der hier zitierten Arbeit ist zu beachten, dass sie sich nicht mit dem in $\$ 6$ Abs. 2a BWahlG 2011 verankerten Modus der Reststimmenverwertung beschäftigt, sondern mit einer früheren, hinsichtlich NSG-Reduzierung weniger geeigneten Version derselben. Insofern sind einige der dort mitgeteilten Ergebnisse und Schlussfolgerungen für das neue BWahlG 2011 nicht zutreffend.

24 Im BWahlG 2011 wird die Fünfprozentsperrklausel weiterhin bundesweit berechnet und nicht individuell für jedes Land. Auch die Reststimmenverwertung operiert länderübergreifend. Durch diese Regelungen bleibt der unitarische Charakter einer Bundestagswahl erhalten und das Wahlgebiet Bundesrepublik zerfällt nicht in ein Nebeneinander der Bundesländer als vollständig separate Teilwahlgebiete. 
Quotient ${ }^{25}$ von der Zweitstimmenzahl abhängt, etabliert konstruierbare Konstellationen, die NSG herbeiführen. Ursächlich dafür ist die Division der aggregierten positiven Reststimmenzahlen geteilt durch den Bundes-Hare-Quotienten zur Errechnung der ReststimmenMandate. Wird der Bundes-Hare-Quotient kleiner, wie es bei fiktiv angenommener Zweitstimmenreduktion der Fall ist, vergrößert sich das Ergebnis der Division. Die Ermöglichung von NSG durch diesen inversen Zusammenhang nebst Rundungsrauschen wird in Abschnitt 3 beispielhaft für zwei Parteien und drei Länder demonstriert.

Gänzlich befreit von NSG wäre der Modus der Reststimmenverwertung dann, wenn statt durch Aggregierung von positiven Reststimmen die Zahl zusätzlicher Mandate durch Aggregierung nebst kaufmännischer Rundung der positiven Sitzbruchteile ${ }^{26}$ für jede Partei ermittelt würde.

\section{Der Regelungsauftrag des $B V \operatorname{erf} G$}

Eine zentrale Fragestellung bei der Bewertung ist es, ob der Gesetzgeber mit dem BWahlG 2011 die Vorgaben des Bundesverfassungsgerichtsurteils hinsichtlich einer Vermeidung, mindestens aber einer weitestgehenden Reduzierung von NSG erfüllt hat. Eine angemessene Beantwortung dieser Frage erfordert eine zweifach differenzierende mathematische Beurteilung. In Tenor Nr. 1 des Urteils hieß es:

„\$7 Absatz 3 Satz 2 (...) verletzt Artikel 38 Absatz 1 Satz 1 des Grundgesetzes, soweit hierdurch ermöglicht wird, dass ein Zuwachs an Zweitstimmen zu einem Verlust an Sitzen der Landeslisten oder ein Verlust an Zweitstimmen zu einem Zuwachs an Sitzen der Landeslisten führen kann. "27

Wortgenau daran angelehnt wird in diesem Aufsatz unter NSG die Fallgestaltung verstanden, dass eine Partei mit hypothetisch mehr Zweitstimmen als den tatsächlich erhaltenen weniger Sitze bekommen hätte beziehungsweise der umgekehrte Fall. Damit ist NSG einerseits ein unilateraler Effekt, an dem nur eine Partei beteiligt ist, andererseits ein monokausaler Effekt.

In der Begründung des Urteils findet sich allerdings bei Randnummer 103 eine Formulierung, die dahingehend interpretierbar ist, dass das BVerfG auch einen bilateralen mandatswirksamen Effekt in die Betrachtung einbezogen hat:

„Ein Wahlsystem, das (...) in typischen Konstellationen zulässt, dass ein Zuwachs an Stimmen zu Mandatsverlusten führt oder dass für den Wahlvorschlag einer Partei insgesamt mehr Mandate erzielt werden, wenn auf ihn selbst weniger oder auf einen konkurrierenden Vorschlag mehr Stimmen entfallen ${ }^{28}$ (...) lässt den demokratischen Wettbewerb um Zustimmung bei den Wahlberechtigten widersinnig erscheinen."

25 Im BWahlG 2011 ( $\$ 6$ Abs. 2a Satz 1) wird dies wörtlich ausgedrückt als die „im Wahlgebiet für einen der zu vergebenden Sitze erforderliche Zweitstimmenzahl.“ Ich interpretiere dies als Quotient aller zuteilungsfähigen Zweitstimmen auf Bundesebene geteilt durch die Gesamtzahl von regulär 598 zu vergebenden Mandaten.

26 Errechnet aus dem Quotienten der Zweitstimmenzahl für eine Partei in einem Land geteilt durch den Landes-Hare-Quotienten nebst Vergleich mit der dieser Partei nach Sainte-Laguë/Schepers zugeteilten Sitzzahl in der Unterverteilung.

27 Hervorhebung hinzugefügt.

28 Hervorhebung hinzugefügt. 
Um diese und ähnliche Situationen von der NSG-Definition des Urteilstenors abzugrenzen, sollen auch diese Effekte als Monotoniefehler begrifflich erfasst werden. Bei Randnummer 103 handelt es sich speziell um einen Bilateralen Monotoniefehler, an dem zwei Parteien beteiligt sind

In den zitierten Passagen spricht das BVerfG einmal explizit von Zweitstimmen, einmal explizit von Stimmen, wobei auch im letzteren Fall aus dem Kontext ableitbar ist, dass Zweitstimmen gemeint sind. Es sind also in den zitierten Passagen allein die Zweitstimmen als variierende Größe benannt, so dass im Umkehrschluss angenommen werden kann, dass alle anderen nicht expressis verbis genannten Größen (einschließlich der Wahlbeteiligungen in den Ländern) konstant zu halten sind bei der Bewertung mandatswirksamer inverser Effekte.

Der Auftrag des BVerfG an den Gesetzgeber bezieht sich zudem auf eine Beseitigung von NSG nicht notwendigerweise bei jedem theoretisch denkbaren Fall, sondern laut Randnummer 99 lediglich für Situationen, die sich an der politischen Wirklichkeit orientieren.

Der den Gesetzgeber bindende Regelungsauftrag des BVerfG ist somit dahingehend zu präzisieren, ob das BWahlG 2011 die Möglichkeit von NSG bei Variation der Zweitstimmenzahl einer Partei in einem Land unter Konstanthaltung aller anderen Größen beseitigt oder doch jedenfalls in wirklichkeitsnahen Fällen nahezu gänzlich reduziert.

In der von Matthias Rossi ${ }^{29}$ formulierten Verfassungsbeschwerde wird dies nicht bedacht (siehe dort Abschnitt D.1). Die Frage, wie die Wahlbeteiligung als im BWahlG 2011 mandatswirksame Größe in mathematische Berechnungen einzubringen ist (ob konstant oder variabel), erweist sich aber als wichtig für die Beurteilung von NSG und dessen Auftretenshäufigkeit. In der von Rossi durchgeführten Analyse wird vorausgesetzt, dass mit fiktiven Zweitstimmenverlusten für eine Partei in einem Land auch Erststimmenverluste einhergehen und zwar in einem Umfang, der für eine Veränderung der Sitzkontingente ausreicht. Diese zusätzlich unterstellten Erststimmenverluste können aber Auswirkungen auf die Zahl der von der Partei errungenen Direktmandate haben. Das aber sind NSG-vermeidende Auswirkungen. Um inverse mandatswirksame Effekte herbeizuführen wird in der genannten Analyse zu Lasten des BWahlG 2011 zwar die potentiell NSG-erzeugende Wirkung der fiktiven Zweitstimmenverluste berücksichtigt, aber die potentiell NSG-vermeidende Wirkung der fiktiven Erststimmenverluste auf die Direktmandatszahl ignoriert, was die Analyse zu Ungunsten des Wahlrechts 2011 verzerrt.

Bei Konstanthaltung der Wahlbeteiligungen kann sich NSG nur durch die Reststimmenverwertung einstellen. Nach Ermittlungen des Bundesministeriums des Inneren ${ }^{30}$ wäre es bei den Wahlergebnissen der Bundestagswahlen 2005 und 2009 bei Anwendung des BWahlG 2011 zu keinem Fall von NSG gekommen. Auch der NSG-Fall im Zusammenhang mit der Dresdner Nachwahl 2005 wäre nicht eingetreten. Dennoch kann NSG im neuen Wahlrecht theoretisch noch bei ungünstigem Zusammenwirken von Einzelfaktoren auftreten. Dies sei der Übersichtlichkeit halber für nur zwei Parteien und drei Länder exemplifiziert (vgl. Tabelle 3).

29 Vgl. Matthias Rossi, Verfassungsbeschwerde gegen das System der Sitzzuteilung im Deutschen Bundestag nach dem geltenden Bundeswahlgesetz, 2011, 2 BvR 2670/11.

30 Genauer das Bundesamt für Sicherheit in der Informationstechnik (BSI), das auf Vermittlung des Bundesministeriums des Inneren nach meinen Vorgaben zusätzliche, sehr detaillierte und große Kapazitäten erfordernde Simulationen der Wahlrechtsgestaltungen durchgeführt hat. 
Insgesamt seien 20 Mandate zu vergeben, 130 Wähler nehmen an der Wahl teil. Angenommen, die Erst- und Zweitstimmenzahlen für zwei Parteien P, Q und deren Kandidaten in drei Ländern X, Y, Z sind derart, dass Partei Q keine Überhangmandate erzielt hat. Hinsichtlich der Erststimmen werde angenommen, dass jeder Wähler den Kandidaten derselben Partei wie mit seiner Zweitstimme gewählt hat. Die Reststimmen der Parteien seien ferner so gestaffelt, dass die Summation der positiven Werte geteilt durch den Bundes-Hare-Quotienten einen Wert für Partei Q von geringfügig weniger als 0,5 ergibt, so dass die Zuteilung eines Reststimmen-Mandats von Partei Q gerade knapp verpasst wird. Der entsprechende Wert für Partei P sei wesentlich kleiner als 0,5.

Nun werde fiktiv angenommen, dass Partei Q in Land Z geringfügig weniger Zweitstimmen erhalte, bei unveränderter Wahlbeteiligung. Die Oberverteilung bleibt dann unverändert. Die Zweitstimmenreduktion in Land Z sei so gering, dass sich auch an der Unterverteilung nach Sainte-Laguë/Schepers in diesem Land nichts ändere. Durch die Zweitstimmen-Einbuße von Partei Q verringert sich aber der Landes-Hare-Quotient von Land Z sowie auch der Bundes-Hare-Quotient. War die ursprüngliche Anzahl der Reststimmen in Land Z für Partei Q negativ, so ist sie es auch nach der Herabsetzung der Zweitstimmenzahl. Die Summe aller positiven Reststimmen ändert sich für Partei Q also nicht. Jedoch wird diese Summe nun durch einen kleineren Bundes-Hare-Quotienten geteilt. Ist dieser mindestens so klein, dass die Division der Summe positiver Reststimmen geteilt durch den Bundes-Hare-Quotienten nunmehr größer als 0,5 ist, so wird dieser Wert aufgerundet und nicht wie vorher abgerundet. Für Partei Q ergibt sich somit bei geringerer Zweitstimmenzahl in diesem Wirkungsgefüge ein zusätzliches Reststimmen-Mandat. Da nach getroffener Annahme keine Überhangmandate für Partei Q vorliegen, wird dieses zusätzliche Mandat zugeteilt.

Allgemeiner würde sich das in obigem Beispiel Demonstrierte ergeben, wenn die Zahl der Überhangmandate von Partei Q der Zahl ihrer Reststimmen-Mandate vor der fiktiven Zweitstimmenreduktion höchstens gleich ist. Dies ist qualitativ das einzige Grundszenario, bei dem sich NSG im BWahlG 2011 ergeben kann. Im BWahlG 2008 stellen Überhangmandate eine notwendige, aber noch nicht hinreichende Bedingung für NSG dar. Je mehr Überhangmandate es gibt, desto größer ist die Wahrscheinlichkeit, dass NSG auftritt. Dieser Zusammenhang wurde im BWahlG 2011 aufgebrochen. Für dieses Wahlrecht ist der umgekehrte wahrscheinlichkeitstheoretische Zusammenhang gültig: Je mehr Überhangmandate es gibt, desto geringer ist die Wahrscheinlichkeit, dass NSG auftritt.

Das Bundesamt für Sicherheit in der Informationstechnik (BSI) hat mit einer MonteCarlo-Methode hypothetische Wahlergebnisse simuliert, die im Umfeld der tatsächlichen Wahlausgänge der Bundestagswahlen 2005 und 2009 liegen. Es wurde ein Simulationskorridor von plus/minus 20 Prozent um die tatsächlichen Wahlergebnisse gewählt. In diesem Korridor wurden zunächst je 1.000 realistische Wahlergebnisse generiert, die somit jeweils in der Nähe der beiden letzten Bundestagswahlergebnisse angesiedelt sind. Dabei wurden - entgegen teils vorgebrachter Kritik ${ }^{31}$ - auch die Wahlbeteiligungen in den Ländern variiert. Um hinreichende Realitätsnähe zu gewährleisten, wurden darüber hinaus in die zufällig erzeugten Wahlergebnisse die folgenden drei Voraussetzungen quantitativ eingearbeitet:

31 Siehe etwa Matthias Rossi, a.a.O. (Fn. 29), S. 15; Friedrich Pukelsheim, Nachtrag zur BMI-Tischvorlage (A-Drs. 17(4) 331), 2011, Ausschuss-Drucksache 17(4) 334, S. 2. 


\begin{tabular}{|c|c|c|c|c|c|c|}
\hline \multicolumn{7}{|c|}{$\begin{array}{l}\text { Tabelle 3: Beispielrechnung für eine Situation mit NSG durch Reststimmenverwertung nach } \\
\text { BWahlG } 2011\end{array}$} \\
\hline & \multicolumn{2}{|r|}{ Land X } & Land Y & \multicolumn{2}{|c|}{ Land Z } & Summe \\
\hline Partei P & \multicolumn{2}{|r|}{5} & 30 & \multicolumn{2}{|c|}{15} & 50 \\
\hline Partei Q & \multicolumn{2}{|r|}{25} & 15 & \multicolumn{2}{|c|}{40} & 80 \\
\hline Wählerzahl & \multicolumn{2}{|r|}{30} & 45 & \multicolumn{2}{|c|}{55} & 130 \\
\hline \multicolumn{7}{|c|}{ Nach Sainte-Laguë/Schepers werden nun Oberverteilung und Unterverteilung berechnet. } \\
\hline & \multicolumn{2}{|c|}{ Land X } & Land Y & \multicolumn{2}{|c|}{ Land Z } & Summe \\
\hline Partei P & \multicolumn{2}{|r|}{1} & 5 & \multicolumn{2}{|l|}{2} & 8 \\
\hline Partei Q & \multicolumn{2}{|r|}{4} & 2 & \multicolumn{2}{|l|}{6} & 12 \\
\hline Oberverteilung & \multicolumn{2}{|r|}{5} & 7 & \multicolumn{2}{|l|}{8} & 20 \\
\hline \multicolumn{7}{|c|}{ Vorstehend sind die Reststimmen-Mandate noch nicht berücksichtigt. Diese werden nun ermittelt. } \\
\hline & Land X & Land Y & Land Z & \begin{tabular}{|c|} 
Summe \\
positiver \\
Reststimmen
\end{tabular} & Quotient & $\begin{array}{c}\text { Rest- } \\
\text { stimmen- } \\
\text { Mandate }\end{array}$ \\
\hline Partei P & $-1,000$ & $-2,143$ & 1,250 & 1,250 & 0,192 & 0 \\
\hline Partei Q & 1,000 & 2,143 & $-1,250$ & \multirow[t]{2}{*}{3,143} & 0,484 & 0 \\
\hline Landes-Hare-Quotient & 6,000 & 6,429 & 6,875 & & & \\
\hline $\begin{array}{l}\text { In der Spalte "Quotient } \\
\text { ten von } 130: 20=6,5 \mathrm{~g} \\
\text { erhalten. } \\
\text { Es werde nun angenomn } \\
40 \text { Erststimmen. }\end{array}$ & $\begin{array}{l}\text { ird die Su } \\
\text { ilt. Die let } \\
\text {, dass Part }\end{array}$ & $\begin{array}{l}\text { umme der po } \\
\text { etzte Spalte te } \\
\text { rtei Q in Lanc }\end{array}$ & $\begin{array}{l}\text { ven Reststi } \\
\text { mit, dass w } \\
\text { statt } 40 \mathrm{nu}\end{array}$ & $\begin{array}{l}\text { nmen durch de } \\
\text { eder P noch Q } \\
35 \text { Zweitstimn }\end{array}$ & $\begin{array}{l}\text { Bundes- } H \\
\text { in Reststim } \\
\text { nen erhält, a }\end{array}$ & $\begin{array}{l}\text { Iare-Quotien- } \\
\text { men-Mandat } \\
\text { ber weiterhin }\end{array}$ \\
\hline & & Land X & Land Y & Land & & Summe \\
\hline Partei P & & 5 & 30 & 15 & & 50 \\
\hline Partei Q & & 25 & 15 & 35 & & 75 \\
\hline Wählerzahl & & 30 & 45 & 50 & & 125 \\
\hline $\begin{array}{l}\text { Der Landes-Hare-Quot } \\
\text { nunmehr } 125: 20=6,2 \\
\text { Partei Q von } 0,484 \text { auf }\end{array}$ & $\begin{array}{l}\text { in Land } \\
\text { amit erh } \\
\text { 3, so das }\end{array}$ & $\begin{array}{l}\mathrm{d} \mathrm{Z} \text { und der } \\
\text { höht sich in } \mathrm{d} \\
\text { sss für diese } \mathrm{P} \text {. }\end{array}$ & $\begin{array}{l}\text { des-Hare } \\
\text { it „Quoti } \\
\text { ein Rests }\end{array}$ & $\begin{array}{l}\text { Quotient änder } \\
\text { nt" überschrieb } \\
\text { immen-Manda }\end{array}$ & $\begin{array}{l}\text { n sich. Let } \\
\text { enen Spalte } \\
\text { resultiert. }\end{array}$ & $\begin{array}{l}\text { terer beträgt } \\
\text { ler Wert von }\end{array}$ \\
\hline & Land X & Land $Y$ & Land Z & \begin{tabular}{|c|} 
Summe \\
positiver \\
Reststimmen
\end{tabular} & Quotient & $\begin{array}{c}\text { Rest- } \\
\text { stimmen- } \\
\text { Mandate }\end{array}$ \\
\hline Partei P & $-1,000$ & $-2,143$ & 2,500 & 2,500 & 0,400 & 0 \\
\hline Partei Q & 1,000 & 2,143 & $-2,500$ & 3,143 & 0,503 & 1 \\
\hline Landes-Hare-Quotient & 6,000 & 6,429 & 6,250 & & & \\
\hline
\end{tabular}

(1) Zweitstimmenverluste einer Partei (relativ zum tatsächlichen Wahlergebnis) hängen in aller Regel mit Zweitstimmengewinnen bei den anderen Parteien zusammen.

(2) Das Wählerverhalten im gesamten Wahlgebiet differiert in den verschiedenen Ländern (relativ zum tatsächlichen Wahlergebnis) nicht zusammenhanglos.

(3) Zweitstimmenzahlen und Erststimmenzahlen für eine Partei beziehungsweise deren Kandidaten variieren (relativ zum tatsächlichen Wahlergebnis) nicht zusammenhanglos.

Die Erzeugung realitätsnaher, alternativer Wahlergebnisse und die durchgeführten Analysen dienen dem Zweck einer wahrscheinlichkeitstheoretischen Quantifizierung von NSG in diversen Wahlrechtsmodellen. 
Jedes erzeugte alternative Wahlergebnis aus einem realistischen Umfeld der vorausgegangenen Bundestagswahlen wurde sodann auf NSG untersucht. Es ist wichtig zu vermerken, dass es sich bei den am BSI vorgenommenen Rechnungen um ceteris-paribus-Simulationen handelt. Mit diesem Terminus wird ausgedrückt, dass dabei alle auf die Sitzzuteilung potentiell einwirkenden Einflussgrößen konstant gehalten werden bis auf eine einzige, deren quantitativer Einfluss herausgearbeitet werden soll, eben die Zweitstimmenzahl für jeweils eine Partei in jeweils einem Land.

Das erweist sich als ein für die Beurteilung der NSG-Wahrscheinlichkeit entscheidender Punkt. Unter Anwendung des BWahlG 2011 kann es auch bei Variation der Wahlbeteiligung (selbst ohne Variation der Zweitstimmenzahlen) in einem einzigen Land in Sonderfällen zu inversen Variationen bei der Gesamtzahl der zugeteilten Mandate auf Bundesebene kommen. Dieser Umstand kann sich dahingehend realisieren, dass eine Verminderung der Zahl der abgegebenen Wahlzettel in seltenen Szenarien zu einer Zunahme der bundesweit insgesamt zugeteilten Mandate führt.

Das BVerfG hat allein auf den mandatswirksamen inversen Effekt bei Variation der Zweitstimmen Bezug genommen. Damit sich beide theoretisch möglichen Effekte - der mandatszahländerne inverse Effekt sowohl bei Variation der Wählerstimmen in einem Land als auch bei Variation der Zweitstimmen für eine Partei in einem Land - nicht überlagern, vielmehr eindeutig trennbar bleiben und Wirkungen kausal zugeordnet werden können, ist es nötig, zur Bestimmung der Möglichkeiten und Wahrscheinlichkeiten des Auftretens von NSG die Wählerzahlen konstant zu halten. Darauf beziehen sich die in den Tabellen 4 bis 6 zusammengefassten Ergebnisse. Zusätzlich wurden aber vom BSI nach meinen Vorgaben auch für mitvariierende Wählerzahlen Simulationen durchgeführt, um Bikausale Monotoniefehler wahrscheinlichkeitstheoretisch zu quantifizieren. Die Ergebnisse sind dokumentiert in Tabelle 7 .

Tabelle 4 ist wie folgt zu interpretieren: Bei jedem der für 2005 und 2009 jeweils generierten 1.000 zufälligen Wahlergebnisse gibt es eine maximale Zahl von 160 NSG Fällen (fünf Parteien in jedem der 16 Länder ergeben 80 betroffene Landeslisten, und für jede dieser Landeslisten besteht die Möglichkeit von NSG bei fiktivem Zweitstimmen-Zuwachs oder -Schwund). Bei den 1.000 simulierten Wahlergebnissen im Umfeld der Wahl 2005 kam es unter Anwendung des BWahlG 2008 bei jedem einzelnen Wahlergebnis mindestens einmal zu NSG. Der Durchschnitt errechnete sich zu 9,73 Fällen je generiertes Wahlergebnis. Das konkurrierende SPD-Modell zeigte noch in 776 von 1.000 Wahlergebnissen NSG und reduzierte die durchschnittliche Zahl der Fälle auf 7,63. Die auftretenden Häufigkeiten für NSG sind für das SPD-Modell im Vergleich mit dem BWahlG 2008 immer noch be-

\section{Tabelle 4: Simulationen zum Negativen Stimmgewicht bei einfacher Zählung}

\begin{tabular}{|c|c|c|c|c|c|c|c|c|c|c|c|c|}
\hline \multirow[b]{2}{*}{ Wahl } & \multicolumn{4}{|c|}{ BWahlG 2008} & \multicolumn{4}{|c|}{ BWahlG 2011} & \multicolumn{4}{|c|}{ SPD-Modell } \\
\hline & $\begin{array}{c}\text { Simu- } \\
\text { lations- } \\
\text { läufe mit } \\
\text { NSG }\end{array}$ & Min & Max & $\begin{array}{c}\text { Mittel- } \\
\text { wert }\end{array}$ & $\begin{array}{c}\text { Simu- } \\
\text { lations- } \\
\text { läufe mit } \\
\text { NSG }\end{array}$ & Min & Max & $\begin{array}{c}\text { Mittel- } \\
\text { wert }\end{array}$ & $\begin{array}{c}\text { Simu- } \\
\text { lations- } \\
\text { läufe mit } \\
\text { NSG }\end{array}$ & Min & Max & $\begin{array}{c}\text { Mittel- } \\
\text { wert }\end{array}$ \\
\hline 2005 & 1.000 & 2 & 18 & 9,73 & 6 & 0 & 8 & 0,03 & 776 & 0 & 38 & 7,63 \\
\hline 2009 & 1.000 & 1 & 20 & 8,80 & 19 & 0 & 10 & 0,07 & 619 & 0 & 53 & 5,93 \\
\hline
\end{tabular}

Quelle: Am BSI durchgeführte Simulationen. 


\begin{tabular}{|c|c|c|c|c|c|c|c|c|}
\hline \multirow[b]{2}{*}{ Wahl } & \multicolumn{4}{|c|}{ BWahlG 2011} & \multicolumn{4}{|c|}{ SPD-Modell } \\
\hline & $\begin{array}{c}\text { Simulationsläufe } \\
\text { mit NSG }\end{array}$ & Min & Max & Mittelwert & $\begin{array}{c}\text { Simulationsläufe } \\
\text { mit NSG }\end{array}$ & Min & Max & Mittelwert \\
\hline 2005 & 6 & 0 & 8 & 0,03 & 776 & 0 & 41 & 9,77 \\
\hline 2009 & 19 & 0 & 10 & 0,07 & 619 & 0 & 56 & 6,19 \\
\hline
\end{tabular}

achtlich. Bei Anwendung des BWahlG 2011 gibt es NSG lediglich noch in sechs von 1.000 Simulationsläufen und der Mittelwert liegt bei 0,03. Zwar ist der Effekt damit nicht mit mathematischer Notwendigkeit ausgeschlossen, aber er tritt nur noch mit einer Wahrscheinlichkeit in der Ein-Prozent-Größenordnung auf.

Im Normalfall wächst der Graph der mathematischen Funktion, die der Zweitstimmenzahl einer Partei in einem Land die Zahl der bundesweiten Mandate zuordnet, monoton nach Art einer Treppe. Im Falle von NSG tritt in diesem funktionalen Zusammenhang mindestens eine Stelle auf, an der der Graph in einem kurzen Intervall monoton fallend ist. Bei einfacher Zählung (vgl. Tabelle 4) werden die von mindestens einer Monotoniestörung betroffenen Landeslisten gezählt. Es könnten theoretisch aber auch mehrere Stellen des Graphen für eine gegebene Landesliste von dieser Monotoniestörung betroffen sein, also mehrfach NSG auftreten. Bei mehrfacher Zählung (vgl. Tabelle 5) werden alle Fälle von NSG separat gezählt. Mit diesen zusätzlichen Daten zur NSG-Intensität kann eingeschätzt werden, ob NSG häufiger als nur in seltenen Ausnahmefällen auftritt.

Die Intensitätszahlen von NSG, repräsentiert durch die Mittelwerte in Tabelle 5, sind für das BWahlG 2011 bei mehrfacher Zählung gegenüber einfacher Zählung nur der betroffenen Landeslisten unverändert. Es ist berechtigt, bei diesen verbliebenen Instanzen von „seltenen Ausnahmefällen“ zu sprechen.

Tabelle 6 widmet sich Bilateralen Monotoniefehlern bei einfacher Zählung. Bei mehrfacher Zählung bleiben die Einträge für das BWahlG 2011 natürlich unverändert bei 0, für das SPD-Modell erhöhen sich die Mittelwerte von 8,56 auf 17,67 und von 5,75 auf 13,22.

Als Fazit ergibt sich der Befund, dass das BWahlG 2011 diese Spielart von Monotoniefehlern mit an Sicherheit grenzender Wahrscheinlichkeit beseitigt. Beim SPD-Modell sind entsprechende Mittelwerte für diesen Monotoniefehler gegenüber dem NSG-Szenario erhöht, darauf hinweisend, dass dieser Monotoniefehler somit stärker ausgeprägt ist.

\begin{tabular}{|c|cccc|cccc|}
\hline \multicolumn{2}{|c|}{ Tabelle 6: Simulationen zum Bilateralen Monotoniefehler (laut Rn. 103, BVerfG-Urteil 2008) } \\
bei einfacher Zählung \\
\hline \multirow{3}{*}{ Wahl } & \multicolumn{3}{|c|}{ BWahlG 2011 } & \multicolumn{4}{c|}{ SPD-Modell } \\
\cline { 2 - 9 } & $\begin{array}{c}\text { Simulationsläufe } \\
\text { mit Monotoniefehler }\end{array}$ & Min & Max & $\begin{array}{c}\text { Mittel- } \\
\text { wert }\end{array}$ & $\begin{array}{c}\text { Simulationsläufe } \\
\text { mit Monotoniefehler }\end{array}$ & Min & Max & $\begin{array}{c}\text { Mittel- } \\
\text { wert }\end{array}$ \\
\hline 2005 & 0 & 0 & 0 & 0 & 646 & 0 & 30 & 8,56 \\
2009 & 0 & 0 & 0 & 0 & 601 & 0 & 20 & 5,75 \\
\hline
\end{tabular}

Anmerkung: Bilaterale Monotoniefehler treten im BWahlG 2008 nicht auf. Die zugehörigen Werte sind also jeweils 0 .

Quelle: BSI-Simulationen nach Vorgaben des Verfassers. 


\begin{tabular}{|c|c|c|c|c|}
\hline \multicolumn{3}{|c|}{ Tabelle 7: Simulationen zum Bikausalen Monotoniefehler bei einfacher Zählung } \\
\hline \multirow{2}{*}{ Wahl } & \multicolumn{4}{|c|}{ Bundeswahlgesetz 2011 } \\
\cline { 2 - 5 } & $\begin{array}{c}\text { Simulationsläufe } \\
\text { mit Bikausalen } \\
\text { Monotoniefehlern }\end{array}$ & Min & Max & Mittelwert \\
\hline 2005 & 994 & 0 & 25 & 9,42 \\
2009 & 970 & 0 & 24 & 7,09 \\
\hline
\end{tabular}

Monotoniefehler höherer Ordnung oder verwandter Artung wurden wegen zu großer inhaltlicher Divergenz zur NSG-Definition im Bundesverfassungsgerichtsurteil von 2008 nicht in die Analyse einbezogen.

Zusammenfassend ergibt sich der Befund, dass durch das BWahlG 2011 die NSG-Problematik im Sinne des genannten Tenors des BVerfG-Entscheids und auch die Problematik des dort in Rn. 103 angesprochenen Bilateralen Monotoniefehlers als hinreichend behoben angesehen werden kann.

Die Simulationsergebnisse zum Bikausalen Monotoniefehler sind in Tabelle 7 erfasst.

Im BWahlG 2008 und im SPD-Modell tritt die Unterscheidung von NSG und Bikausalem Monotoniefehler nicht auf. Für das BWahlG 2011 schließen die verbleibenden Bikausalen Monotoniefehler die Situation der Nachwahl 2005 im Dresdner Wahlkreis 160 ein, die als Auslöser für das Urteil des BVerfG vom 3. Juli 2008 fungierte. Wären in diesem Wahlkreis 5.000 CDU-Wähler der Wahl fern geblieben (also unter der weitergehenden Annahme, dass diese neben ihrer Zweitstimme auch ihre Erststimme entziehen, so dass zusätzlich zu einem Schwund von 5.000 Zweitstimmen bei der CDU sich die Gesamtzahl der Wählerstimmen in Sachsen um 5.000 Stimmen reduziert), so hätte bei Anwendung des BWahlG 2011 dies wegen der Überhangsituation die Zahl der CDU-Sitze in Sachsen unverändert belassen, in Berlin wäre der CDU dagegen ein weiterer Sitz zuteil geworden (Bikausaler Monotoniefehler). Dagegen steht: Bei alleiniger Abnahme der Zweitstimmen der CDU im Wahlkreis 160 um 5.000 Stimmen ohne gleichzeitige Reduktion der Wählerstimmenzahl in Sachsen (etwa indem die den Zweitstimmen zugeordneten Erststimmen von den Wählern unverändert abgegeben worden wären) kommt es nicht zu einem Mandatsgewinn der CDU auf Bundesebene und folglich nicht zu NSG.

Das bedeutet aber, dass unter dem BWahlG 2011 die Nachwahl im Dresdner Wahlkreis politisch völlig anders verlaufen wäre als unter dem BWahlG 2008. Während die CDU bei der Nachwahl alle ihre Anhänger sinnvoll aufrufen konnte, mit ihrer Zweitstimme nicht CDU zu wählen, würde unter dem BWahlG 2011 dieser Aufruf für sich allein der CDU gar nichts nützen. Um etwas zu erreichen, hätte die CDU zudem 5.000 ihrer Anhänger aufrufen müssen, auch mit der Erststimme nicht den CDU-Kandidaten zu wählen, sondern sie gar nicht abzugeben, um die benötigte Verschiebung der Ländersitzkontingente zu erreichen. Das aber ist kein politisch sinnvoller Aufruf an die eigene Anhängerschaft, denn wenn zu viele ihrer Anhänger darauf eingehen, verliert die CDU das Direktmandat. Ihr Kandidat hatte nur 7.600 Erststimmen Vorsprung. Unter dem BWahlG 2011 hätte die CDU also im Wahlkampf ihre Anhänger nicht aufgerufen, mit der Zweitstimme nicht die CDU zu wählen, während die SPD empfahl, genau dies zu tun. Der Wahlkampf wäre nicht invers, sondern normal verlaufen. 
Hinzu kommt, dass Bikausale Monotoniefehler, sieht man vom seltenen Fall einer Nachwahl ab, nur in sehr schmalen Fenstern von Stimmenzahlen auftreten. Die Berechnungen von Ulrich Wiesner ${ }^{32}$ bestätigen dies. Zudem sind bundesweit höchstens ein bis zwei Mandate betroffen. Ferner ist die Lage des die Monotonie störenden Intervalls im zuvor angesprochenen mathematischen Graphen vom Zufall abhängig. Nur in einer ex-post Analyse nach der Wahl ist feststellbar, ob, wo und in welchem Umfang sich Wirkungen Bikausaler Monotoniefehler hätten ergeben können. Aus diesen Gründen sind derartige Monotoniestörungen ex-ante nicht instrumentalisierbar und wegen ihrer geringen Größenordnung nicht in der Lage, selbst kleine Sitzkontingente manipulativ zu verschieben.

\section{Alternative Modelle}

Neben der Koalition aus CDU/CSU und FDP, deren Gesetzentwurf am 29. September 2011 vom Bundestag verabschiedet wurde, hatten auch die Fraktionen der SPD, von Bündnis 90/ Die Grünen und Die Linke eigene Entwürfe zur Novellierung des BWahlG 2008 eingebracht. Auch auf diese alternativen Modelle soll kurz eingegangen werden.

\subsection{Das Modell der SPD-Fraktion}

Der Gesetzentwurf der SPD ${ }^{33}$ sieht die folgenden Schritte bei der Sitzzuteilung vor: In einem ersten Schritt werden die 598 regulären Ausgangssitze des Bundestages nach SainteLaguë/Schepers auf die Parteien im Verhältnis der Zweitstimmen zugeteilt (Oberzuteilung) und anschließend für jede Partei mit demselben Verfahren an die jeweiligen Landeslisten weitergeleitet (Unterzuteilung). Das ist eine vorläufige Sitzzuteilung.

Sofern bei dieser Anfangsanpassung Überhangmandate anfallen, wird im zweiten Schritt von 598 Sitzen ausgehend die Gesamtzahl der zu vergebenden Sitze so lange schrittweise um jeweils einen erhöht und die Oberzuteilung neu berechnet, bis jede Partei mindestens so viele Sitze zugeteilt bekommt wie im Anfangsschritt bei der ursprünglichen Oberzuteilung zuzüglich ihrer Überhangmandate. Das ist die Zwischenanpassung.

Schließlich folgt die Endanpassung: Bei der abermaligen Unterzuteilung wird die Zahl der Sitze aus der Zwischenanpassung nach dem Augsburger Zuteilungsverfahren so an die Landeslisten verteilt, dass jede mindestens so viele Mandate erhält, wie Kandidaten Direktmandate zu ihren Gunsten erzielt haben.

Das Modell hat den Vorteil, dass alle Wahlkreisgewinner in den Bundestag einziehen und gleichzeitig die an die Parteien vergebenen Sitze im Verhältnis der von ihnen errungenen Zweitstimmen zugeteilt werden. Die Erfolgswertgleichheit der Wählerstimmen ist auf Bundesebene in dem Maße gesichert, wie es das diesbezüglich bestmögliche Verfahren von Sainte-Laguë/Schepers gewährleisten kann.

Das Modell hat einen Nachteil, der generell Zuteilungsmodellen zukommt, die Ausgleichsmandate für Überhangmandate vergeben, um diese zu kompensieren. Er betrifft

32 Siehe Ulrich Wiesner, Sitzzuteilungsverfahren, http://ulrichwiesner.de/stimmgewicht/ (Abruf am 17. Januar 2012).

33 BT-Drucksache 17/5895 vom 24. Mai 2011. 


\begin{tabular}{|c|c|c|c|c|c|c|c|c|}
\hline & $\begin{array}{l}\vec{\Xi} \\
\tilde{\tilde{J}} \\
\tilde{u}\end{array}$ & 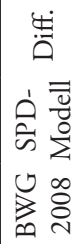 & 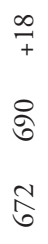 & 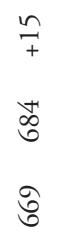 & \begin{tabular}{l} 
m \\
\multirow{8}{0}{} \\
$\stackrel{+}{0}$
\end{tabular} & 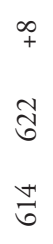 & 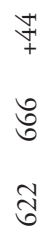 & 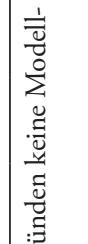 \\
\hline & 艺 & 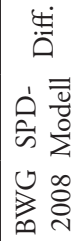 & $\mp$ & $\begin{array}{l}F \\
\hat{n} \\
\stackrel{n}{ }\end{array}$ & $\begin{array}{l}\text { N } \\
+1 \\
N\end{array}$ & $\stackrel{\sim}{+}$ & 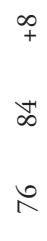 & 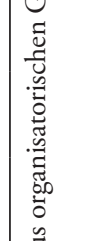 \\
\hline & $\underset{:}{\mathscr{Z}}$ & 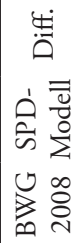 & ๙ิ & $\begin{array}{l}\stackrel{+}{+} \\
\stackrel{+}{ } \\
\underset{F}{F}\end{array}$ & $\begin{array}{l}7 \\
i \\
i n\end{array}$ & $\mp$ & $\begin{array}{l}\stackrel{\infty}{+} \\
\stackrel{\infty}{\gtrless} \\
\infty\end{array}$ & 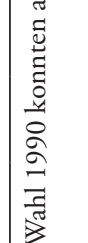 \\
\hline & 宔 & 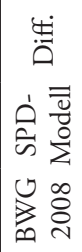 & $\begin{array}{l}\stackrel{+}{+} \\
\stackrel{\circ}{\circ} \\
\stackrel{\leftrightarrow}{*}\end{array}$ & $\begin{array}{l}\stackrel{+}{+} \\
\stackrel{\sim}{f} \\
\stackrel{F}{F}\end{array}$ & $\begin{array}{l}F \\
+ \\
\stackrel{\infty}{+} \\
\stackrel{+}{F}\end{array}$ & \begin{tabular}{l}
$\stackrel{m}{+}$ \\
\multirow{6}{*}{}
\end{tabular} & $\begin{array}{l}\stackrel{0}{+} \\
\approx \\
0 \\
\approx\end{array}$ & 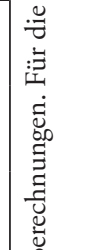 \\
\hline 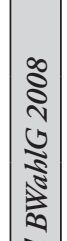 & 2 & 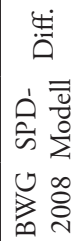 & $\begin{array}{l}\stackrel{+}{+} \\
\stackrel{\sim}{n} \\
\stackrel{n}{n}\end{array}$ & $\begin{array}{l}\stackrel{+}{+} \\
\stackrel{+}{ } \\
\underset{F}{F}\end{array}$ & $\begin{array}{l}\text { +1 } \\
\text { i } \\
\stackrel{\infty}{n}\end{array}$ & $\stackrel{\sim}{+}$ & 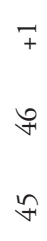 & 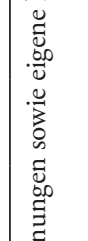 \\
\hline 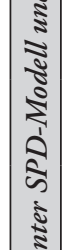 & ? & 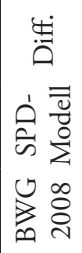 & $\begin{array}{l}\stackrel{0}{+1} \\
\stackrel{\sim}{d} \\
\stackrel{\sim}{\sim}\end{array}$ & $\begin{array}{l}\stackrel{\infty}{+} \\
\stackrel{2}{\curvearrowright} \\
\stackrel{\infty}{\varrho}\end{array}$ & $\begin{array}{l}\mp \\
\approx \\
\Xi\end{array}$ & $\begin{array}{l}\infty \\
\stackrel{\infty}{\infty} \\
\stackrel{\infty}{-}\end{array}$ & $\begin{array}{l}0 \\
+1 \\
\stackrel{+}{2} \\
\stackrel{+}{\beth}\end{array}$ & 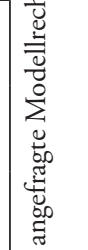 \\
\hline 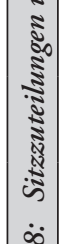 & $\hat{\bar{\omega}}$ & 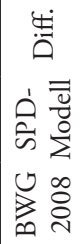 & 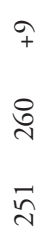 & 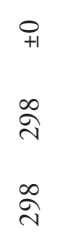 & $\begin{array}{l}\text { +1 } \\
\vec{n} \\
\sqrt{n} \\
\vec{\sim}\end{array}$ & $\begin{array}{l}\text { N } \\
\underset{N}{N}\end{array}$ & 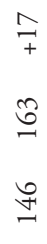 & 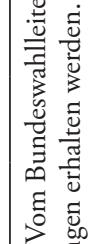 \\
\hline$\frac{\Xi ँ}{\Xi}$ & & $\geqslant$ & $\stackrel{+}{\sigma}$ & $\stackrel{\infty}{\check{\sigma}}$ & ڤิ & 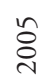 & ڤे & 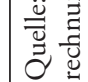 \\
\hline
\end{tabular}




\begin{tabular}{|l|c|c|c|c|c|c|c|}
\hline Tabelle 9: Die Bundestagswahl 2009 unter dem SPD-Modell \\
\hline SPD-Modell & SPD & CDU & CSU & FDP & Grüne & Linke & Gesamt \\
\hline Bundestagswahl 2009 & 163 & 194 & 46 & 103 & 76 & 84 & 666 \\
CDU-Thüringen minus & 164 & 195 & 47 & 104 & 76 & 85 & 671 \\
7.400 Zweitstimmen & & & & & & \\
\hline
\end{tabular}

Anmerkung: Sitzzuteilung unter dem SPD-Modell bei der Bundestagswahl 2009 und bei fiktiv angenommener Zweitstimmenreduktion von 7.400 Stimmen für die CDU in Thüringen.

Quelle: Am BSI durchgeführte Simulationen.

Schwesterparteien und Koalitionspartner: Ein Überhangmandat für eine der Schwesterparteien (CDU) könnte ein oder mehrere Ausgleichsmandate für die andere Schwesterpartei (CSU) nach sich ziehen und so zu einem multiplen Bonus führen. Auch die inverse Situation mit doppeltem Malus ist möglich.

Das Modell hat den weiteren Nachteil, dass es aufgrund der vorgenommenen Strukturänderungen ergebnisseitig nicht mehr nur gering-invasiv ist.

Ein augenfälliges Manko des SPD-Modells ist die ausgeprägte Variabilität der Bundestagsgröße (vgl. Tabelle 8). Im 2009 gewählten Bundestag wären 666 Sitze entstanden. Diese starke Volatilität wird auch in Simulationen bestätigt. Bei numerischen Untersuchungen in einem Umfeld von plus/minus 20 Prozent um das tatsächliche Wahlergebnis von 2009 ergaben sich Bundestagsgrößen von mehr als 900 Sitzen, der Mittelwert lag bei 671 Sitzen.

Diese Simulationen unterstützen auch empirisch den Sachverhalt, dass das SPD-Modell eine sensible Abhängigkeit von geringfügigen Zweitstimmenverschiebungen aufweisen kann: Bei der Bundestagswahl 2009 hätte ein Minus von 7.400 Zweitstimmen für die CDU in Thüringen, bliebe alles andere gleich („,ceteris paribus“), dazu geführt, dass der Bundestag nicht mehr 666, sondern 671 Sitze umfasst hätte. Das ist ein inverser Effekt insofern, als (etwas) weniger abgegebene Zweitstimmen zu einem (in Relation erheblich) vergrößerten Bundestag geführt hätten. Da 7.400 Zweitstimmen etwa einem Zehntel Mandat entsprechen, handelt es sich um eine 50-fache Hebelwirkung. Bei dieser geringfügigen Änderung hätte die CDU zudem statt 194 Sitzen 195 zugeteilt bekommen, so dass auch NSG vorliegt (vgl. Tabelle 9).

Quantifizierende Simulationsergebnisse zur Häufigkeit von NSG beim SPD-Modell wurden bereits in den Tabellen 4 bis 6 präsentiert. Wegen der beschriebenen Eigenschaften ist die Tauglichkeit des SPD-Modells mit einem Fragezeichen zu versehen.

\subsection{Das Modell der Fraktion Bündnis90/Die Grünen}

Der Gesetzentwurf von Bündnis90/Die Grünen ${ }^{34}$ setzt zur Realisierung des BVerfG-Auftrags bei den Überhangmandaten an.

Im ersten Schritt wird die Gesamtzahl der Sitze auf Basis der zuteilungsberechtigten Zweitstimmen nach Sainte-Laguë/Schepers auf die Parteien verteilt (Oberzuteilung). Externe Überhangmandate, die für eine Partei in der Oberzuteilung anfallen, werden durch eine Nichtzuteilungs-Regelung unterbunden. Sie sieht vor, die überzählig gewonnenen Wahl- 
kreissitze an jene Kandidaten dieser Partei mit den geringsten prozentualen Erststimmenanteilen nicht zuzuteilen.

Anschließend werden die einer Partei auf Bundesebene insgesamt zustehenden Mandate mit Sainte-Laguë/Schepers auf die Landeslisten vorläufig verteilt. Dabei werden Mandate, die Direktkandidaten der Partei errungen haben, auf die so ermittelte Sitzzahl angerechnet, interne Überhangmandate bleiben den Parteien erhalten. Anschließend wird der Parteidivisor des Verfahrens von Sainte-Laguë/Schepers so heraufgesetzt, dass die Zahl der dieser Partei zustehenden Sitze unter Berücksichtigung der von ihren Kandidaten erzielten Direktmandate gleich der dieser Partei zukommenden Gesamtsitzzahl ist.

Dieser Gesetzentwurf unterbindet die Zuteilung externer Überhangmandate und kompensiert die in einem Land auftretenden internen Überhangmandate einer Partei durch entsprechende Reduktion der Sitzzahlen bei anderen Landeslisten derselben Partei. Die mögliche verfassungsrechtliche Problematik, die darin besteht, einem direkt gewählten Kandidaten, hinter dem ja ein gewisser Wählerauftrag steht, das Mandat vorzuenthalten, soll hier nicht angesprochen werden. ${ }^{35}$

Der Vorteil dieses Modells ist darin zu sehen, dass der Bundestag stets seine reguläre Größe von 598 Mandaten behält. Auch beseitigt der Entwurf die Möglichkeit von NSG vollständig. Als Nachteil ist zu benennen, dass der föderale Proporz der Zweitstimmen empfindlich beeinträchtigt wird. Nicht nur wird dieser durch die Existenz von Überhangmandaten zugunsten der überhängenden Listen verzerrt (was eine vom BVerfG in früherer Rechtsprechung ${ }^{36}$ tolerierte Verzerrung darstellt), sondern zusätzlich und verstärkend noch durch die komplementär vorgenommenen Kürzungen bei anderen nicht-überhängenden Listen derselben Partei. Länder, die diese Kürzungen hinnehmen müssen, werden doppelt benachteiligt. Gegenüber dem BWahlG 2008 sind die Verwerfungen des föderalen Proporzes demnach quantitativ intensiviert. Faktisch impliziert der Gesetzentwurf eine Benachteiligung von Landeslisten einer Partei mit wenigen Direktmandaten und vielen Zweitstimmen gegenüber solchen Landeslisten mit vielen Direktmandaten, aber wenigen Zweitstimmen.

Die Regelungsteile des Modells zur Handhabung von Überhangmandaten können theoretisch dazu führen, dass eine Landesliste ohne von ihren Kandidaten errungene Direktmandate, die aber hinreichend Zweitstimmen auf sich vereinigt hat, um an sich einen Sitz zu erhalten, diesen aber aus Gründen der Kompensation von anderen überhängenden Landeslisten nicht zugeteilt bekommt, nicht im Bundestag vertreten ist. Konkret wäre bei Anwendung dieses Modells auf das Ergebnis der Bundestagswahl 2009 die CDU in Bremen trotz erreichter 80.964 Zweitstimmen bei einem Bundes-Hare-Quotienten von 68.167,7 nicht mehr im Bundestag vertreten gewesen. ${ }^{37}$

Wie erkennbar führt das Modell zu Veränderungen der Sitzzuteilungszahlen auf Bundesebene lediglich bei den Parteien mit Überhängen. Die Gesamt-Mandate von CDU und CSU werden um deren interne beziehungsweise externe Überhangmandate vermindert (vgl. Tabelle 10).

35 In diesem Kontext relevant ist das 4:4 Urteil des Zweiten Senats des BVerfG zur Frage der Überhangmandate vom 10. April 1997 (2 BvF 1/95, BVerfGE 95, S. 335).

36 Im BVerfGE 95, S. 335 wird die durch Überhangmandate hervorgerufene Proporzverzerrung für verfassungskonform erklärt.

37 Siehe Gerd Strohmeier, Stellungnahme zur Anhörung des Innenausschusses am 5. September 2011, Ausschuss-Drucksache 17(4) 327 H, S. 21 f. 


\begin{tabular}{|l|c|c|c|}
\hline \multicolumn{4}{|c|}{ Tabelle 10: Sitzzuteilungen bei Anwendung des Modells von Bündnis90/Die Grünen und des } \\
BWablG 2008 auf das Ergebnis der Bundestagswabl 2009
\end{tabular}

\subsection{Das Modell der Fraktion Die Linke}

Das Modell der Fraktion Die Linke ${ }^{38}$ setzt wie das Modell von Bündnis90/Die Grünen zur Erfüllung des Regelungsauftrags des BVerfG an den Überhangmandaten an.

Eine Zuteilung der Sitze an die Parteien erfolgt zunächst auf Bundesebene (Oberzuteilung) und dann parteiintern an die Landeslisten der Parteien (Unterzuteilung). In der Oberzuteilung wird die Berechnung der Sitzzahlen nach Sainte-Laguë/Schepers im Verhältnis der zu berücksichtigenden Zweitstimmen vorgenommen, wobei die Fünfprozenthürde abgeschafft ist. Externe Überhangmandate verbleiben den Parteien. In diesem Fall wird die Gesamtzahl der zu vergebenden Sitze um so viele Ausgleichsmandate erhöht, bis auf Bundesebene bei Berücksichtigung der Überhangmandate eine Sitzverteilung im Verhältnis der Zweitstimmenzahlen der Parteien gewährleistet ist.

Die Verteilung der Sitze in der Unterzuteilung an die Landeslisten erfolgt wiederum nach Zweitstimmen mit Sainte-Laguë/Schepers. Treten interne Überhangmandate auf, werden diese durch ebenso viele Listenmandate anderer Landeslisten derselben Partei kompensiert.

Als Vorteil des Entwurfs ist die vollständige Beseitigung von NSG zu sehen. Durch Aufhebung der Fünfprozenthürde wird zwar aus rein mathematischer Perspektive eine größere Erfolgswertgleichheit der Stimmen erreicht, doch ist dies ein gravierender Eingriff, der zum Einzug von Klein- und Kleinstparteien in den Bundestag führt. Ob dieser Eingriff verfassungswidrig ist, kann hier nicht bewertet werden.

Der Gesetzentwurf verzichtet auf die Kappung externer Überhangmandate und bleibt insofern von den damit verbundenen Negativauswirkungen verschont. ${ }^{39}$ Doch ist zu konstatieren, dass mögliche externe Überhangmandate der CSU eine ausgesprochen starke Hebelwirkung entfalten, die quantifizierbar ist: Bei der Bundestagswahl 2009, mit diesem Modell ausgezählt, hätten die drei externen CSU-Überhangmandate zu nicht weniger als 40 Ausgleichsmandaten und einer Bundestagsgröße von 641 Sitzen geführt (vgl. Tabelle 11). Als Negativum ist deshalb zu benennen, dass der Reformvorschlag, vergleichbar dem

38 BT-Drucksache 17/5895 vom 25. Mai 2011.

39 Immer dann, wenn Überhangmandate nicht zugeteilt werden, kann sich die Fragwürdigkeit ergeben, dass ein Wahlkreisgewinner nicht in den Bundestag einzieht (weil sein Mandat der Streichung der Überhangmandate zum Opfer fiel), der von ihm besiegte Wahlkreisverlierer - über die Landesliste seiner Partei - dagegen schon. 


\begin{tabular}{|l|c|c|c|}
\hline \multicolumn{4}{|c|}{ Tabelle 11: Sitzzuteilungen bei Anwendung des Modells der Linken und des BWahlG 2008 auf das } \\
Ergebnis der Bundestagswahl 2009
\end{tabular}

SPD-Modell, zu einer erheblichen, von der Zahl externer Überhangmandate abhängigen Vergrößerung des Bundestages führen kann und deshalb unter praktischen Gesichtspunkten fragwürdig ist. Ferner leidet der Gesetzentwurf durch die Kompensationsregelung für interne Überhangmandate an einer empfindlichen Verzerrung des föderalen Proporzes.

\section{Fazit: Neues Wahlrecht mit Schattenseiten}

Das BVerfG hatte dem Gesetzgeber mit Urteil vom 3. Juli 2008 den Auftrag erteilt, eine verfassungsgemäße Neuregelung des Wahlrechts ohne signifikantes NSG vorzunehmen. Bezieht man sich auf die vom Gericht gegebene Definition von NSG, entspricht das BWahlG 2011 den Vorgaben und ist hinsichtlich Tenor und tragenden Gründen urteilskonform. Auch ist es gegenüber dem im Prinzip bewährten BWahlG 2008 minimal-invasiv. Zwar ist die Novelle nicht frei von Eigenheiten bis hin zu Fragwürdigkeiten und bewirkt Differenzierungen bei Idealanforderungen der Wahlrechtsprinzipien, doch muss bedacht werden, dass es mathematisch unmöglich ist, ein Modell zu konzipieren, das frei von jeglichen Inkonsistenzen ist und allen Idealansprüchen relevanter Verfassungsprinzipien vollständig gerecht wird.

Die mit dem BWahlG 2011 im Bundestag konkurrierenden Entwürfe weisen noch stärkere Mängel auf. Das von der SPD favorisierte Modell ermöglicht substanzielle Fallzahlen von NSG und kann zudem zu einer erheblichen Aufblähung des Bundestages führen. Der Entwurf von Bündnis90/Die Grünen ist zwar frei von NSG, doch kann die Repräsentation der Länder im Parlament unerträglich verzerrt sein. Das Modell Der Linken erzeugt zwar kein NSG, kann jedoch wegen der starken Hebelwirkung externer Überhangmandate den Bundestag erheblich vergrößern. Aufs Ganze gesehen ist das BWahlG 2011 - bei allen Unzulänglichkeiten - hinsichtlich der gemeinsam betrachteten Gesichtspunkte von Fairness, Erfolgswertgleichheit, Unverzerrtheit, Verfassungskonformität und Praktikabilität das Tauglichste unter den genannten Verfahren.

Dennoch erklärte das BVerfG dieses Wahlgesetz mit Urteil vom 25. Juli 2012 für verfassungswidrig. ${ }^{40}$ Die Richter monierten drei Details: Erstens dürfe nicht die Wahlbeteiligung 
darüber entscheiden, wie viele Abgeordnete ein Bundesland in den Bundestag schickt, da diese Festlegung NSG in nicht hinnehmbaren Fallzahlen weiterhin zulasse. Zweitens dürfe bei der Reststimmenverwertung nicht einseitig auf das Rundungspech Bezug genommen werden. Und drittens dürfe ein Wahlrecht nicht mehr als etwa 15 ausgleichslose Überhangmandate zulassen. Im Vergleich zum Entscheid vom 3. Juli 2008 hat das Gericht in diesem jüngsten Wahlrechtsurteil einerseits seine Definition von NSG erweitert und andererseits den Rahmen der Zulässigkeit von Überhangmandaten verkleinert. Es hat also an zwei wichtigen Stellschrauben in einschränkender Weise Änderungen vorgenommen. Damit konnte der Gesetzgeber nicht rechnen. ${ }^{41}$

Inzwischen haben sich die Fraktionen des Bundestages (außer Die Linke) auf ein neues Wahlrecht geeinigt und ein entsprechendes Gesetz noch rechtzeitig vor der nächsten Bundestagswahl verabschiedet. Es handelt sich dabei um eine Kombination aus Anteilen des BWahlG 2011 und dem früheren SPD-Gesetzentwurf. Dieses Kombinationsmodell beinhaltet eine Kompensation von Überhangmandaten durch Ausgleichsmandate. Positiv ist zu werten, dass alle Wahlkreissieger in den Bundestag einziehen. Ferner wird der Proporz der Parteien nach Zweitstimmen im Bundestag gewährleistet. Auch föderale Verzerrungen halten sich in Grenzen. Diese wünschenswerten Eigenschaften sind, von kleineren handwerklichen Mängeln abgesehen, gut realisiert. Das neue Wahlrecht hat aber Schattenseiten. Negativ zu werten ist die starke Volatilität der Bundestagsgröße. In Simulationen ergaben sich Hausgrößen oberhalb von 800 Sitzen. Kleine Veränderungen bei den Stimmenzahlen können zu großen in der Sitzzahl führen. Hätte bei der Bundestagswahl 2009 Die Linke in Hamburg nur 8.000 Stimmen mehr erhalten, würde unter dem neuen Wahlrecht, bliebe alles andere gleich, die Hausgröße von 671 auf 666 reduziert. Das ist wiederum eine rund 50-fache, zudem gegenläufige Hebelwirkung. Dieser Effekt kann sich auch in entgegengesetzter Richtung einstellen. Das Sitzzuteilungsverfahren des Wahlrechts 2013 reagiert demnach mit übergroßer Empfindlichkeit auf kleine Änderungen bei den Stimmenzahlen. Eine weitere Auswirkung der 8.000 zusätzlichen Stimmen für Die Linke in Hamburg bestünde darin, dass diese Partei bundesweit ein Mandat verlöre. Zwar würden auch CDU, SPD, FDP und CSU je ein Mandat einbüßen, doch sinkt neben der Mandatszahl der Linkspartei auch ihr Mandatsanteil. Damit besteht die Möglichkeit von NSG im Sinne des Bundesverfassungsgerichts (und ferner relatives NSG) auch im neuen Wahlrecht.

41 Eine kritische Würdigung des Verfassungsgerichtsurteils wird von Volker M. Haug vorgenommen. Ders., Das Bundesverfassungsgericht als Gesetzgeber anstelle des Gesetzgebers: Ein kritischer Blick auf das Wahlrechtsurteil vom 25. Juli 2012, in: ZParl, 43. Jg. (2012), H. 3, S. 658 - 674. 